SERIE B - INFORMATIK

\title{
Point Sets with Distinct Distances
}

\author{
Hanno Lefmann* \\ Torsten Thiele**
}

B $94-16$

August 1994

\begin{abstract}
For positive integers $d$ and $n$ let $f_{d}(n)$ denote the maximum cardinality of a subset of the $n^{d}$-grid $\{1,2, \ldots, n\}^{d}$ with distinct mutual euclidean distances. Improving earlier results of Erdoss and Guy, it will be shown that $f_{2}(n) \geq c \cdot n^{2 / 3}$ and, for $d \geq 3$, that $f_{d}(n) \geq c_{d} \cdot n^{2 / 3} \cdot(\ln n)^{1 / 3}$, where $c, c_{d}>0$ are constants. Also improvements of lower bounds of Erdös and Alon on the size of Sidon-sets in $\left\{1^{2}, 2^{2}, \ldots, n^{2}\right\}$ are given.

Furthermore, it will be proven that any set of $n$ points in the plane contains a subset with distinct mutual distances of size $c_{1} \cdot n^{1 / 4}$, and for point sets in general position, i.e. no three points on a line, of size $c_{2} \cdot n^{1 / 3}$ with constants $c_{1}, c_{2}>0$. To do so, it will be shown that for $n$ points in $\mathbf{R}^{2}$ with distinct distances $d_{1}, d_{2}, \ldots, d_{t}$, where $d_{i}$ has multiplicity $m_{i}$, one has $\sum_{i=1}^{t} m_{i}^{2} \leq c \cdot n^{3.25}$ for a positive constant $c$. If the $n$ points are in general position, then we prove $\sum_{i=1}^{t} m_{i}^{2} \leq c \cdot n^{3}$ for a positive constant $c$ and this bound is tight. This confirms a conjecture of Erdös and Fishburn.

Moreover, we give an efficient sequential algorithm for finding a subset of a given set with the desired properties, for example with distinct distances, of size as guaranteed by the probabilistic method under a more general setting.
\end{abstract}

\footnotetext{
*Universität Dortmund, Fachbereich Informatik, LS II, D-44221 Dortmund, Germany.

**Freie Universität Berlin, Institut für Mathematik II, Arnimallee 3, D-14195 Berlin, Germany.

E-mail: thiele@math.fu-berlin.de
} 


\section{Introduction}

In [EG] Erdös and Guy considered the following problem: Determine the maximum size of a subset $X$ of the $n \times n$-grid, that is the set $\{1,2, \ldots, n\} \times\{1,2, \ldots, n\}$, such that all mutual euclidean distances between different points of $X$ are distinct, compare also [Gu]. Denoting the cardinality of such a set $X$ by $f_{2}(n)$, they proved the following:

Theorem 1 [EG] For every integer $n \geq 3$,

$$
n^{\frac{2}{3}-\frac{c_{1}}{\ln \ln n}} \leq f_{2}(n) \leq c_{2} \cdot \frac{n}{(\ln n)^{1 / 4}},
$$

where $c_{1}, c_{2}>0$ are constants.

To obtain the lower bound for $f_{2}(n)$, Erdös and Guy used Greedy-type arguments. The upper bound for $f_{2}(n)$ follows from a result of Landau [La], namely, that the number of integers less than $x$, which are representable as a sum of two squares, is asymptotically $c \cdot \frac{x}{(\ln x)^{1 / 2}}$, where $c$ is a positive constant.

Recently, in [Th] by using more refined counting techniques, the lower bound from (1) has been improved:

Theorem 2 [Th] For all integers $n \geq 2$,

$$
f_{2}(n) \geq c \cdot \frac{n^{2 / 3}}{(\ln n)^{1 / 3}},
$$

where $c>0$ is a constant.

In this paper, we will further improve the lower bound on $f_{2}(n)$ by using uncrowded hypergraphs, cf. [AKPSS] and [ALR], as well as some results from number theory, namely we will show:

Theorem 3 For integers $n \geq 1$,

$$
f_{2}(n) \geq c \cdot n^{2 / 3}
$$

where $c>0$ is a constant.

In order to prove this, we will show a so-called anti-Ramsey theorem, which will be given in Section 2. This anti-Ramsey result, together with some number theoretic results which we deduce in Section 3, also yields lower bounds for the analog of the problem of Erdös and Guy in higher dimensions. Let $f_{d}(n)$ denote the maximum size of a subset $X$ of the $d$-dimensional grid $\{1,2, \ldots, n\}^{d}$ such that all mutual euclidean distances within $X$ are distinct.

We remark that for $d=1$ one has $f_{1}(n)=\theta(\sqrt{n})$ by using perfect difference sets, cf. [EG]. For $d \geq 3$, Erdős and Guy showed the following:

Theorem 4 [EG] Let $d \geq 3$ be a positive integer and $\epsilon>0$ a fixed real. Then, for positive integers $n$ sufficiently large,

$$
n^{2 / 3-\epsilon} \leq f_{d}(n) \leq c \cdot \sqrt{d} \cdot n,
$$

where $c>0$ is a constant. 
Indeed, in $[\mathrm{EG}]$ it has been conjectured that

$$
f_{d}(n) \leq c \cdot d^{2 / 3} \cdot n^{2 / 3} \cdot(\ln n)^{1 / 3}
$$

for $d \geq 3$.

In Section 4 we improve the lower bound $(2)$ on $f_{d}(n)$ :

Theorem 5 Let $d \geq 3$ be a positive integer. Then for every integer $n \geq 1$,

$$
f_{d}(n) \geq c_{d} \cdot n^{2 / 3} \cdot(\ln n)^{1 / 3},
$$

where $c_{d}>0$ is a constant only dependent on $d$.

In Section 5 we will consider the corresponding selection problems for points in the plane in arbitrary position. We will show that every $n$-point set in the euclidean plane $\mathbf{R}^{2}$ contains a subset $X$ with mutual distinct distances such that $|X| \geq c \cdot n^{1 / 4}$ for some constant $c>0$. This improves a former result from [Th], where the lower bound $c \cdot n^{2 / 9}$ has been shown. Moreover, we will show that under the assumption that the $n$ points are in general position (no three on a line) the lower bound on $|X|$ can be improved to $c \cdot n^{1 / 3}$. To do so we will prove a conjecture of Erdös and Fishburn [Fi], [EF]. Namely, we will show the following: if $n$ points in general position in $\mathbf{R}^{2}$ are given with distinct distances $d_{1}, d_{2}, \ldots, d_{t}$, where $d_{i}$ occurs with multiplicity $m_{i}, i=1,2, \ldots, t$, then $\sum_{i=1}^{t} m_{i}^{2} \leq c \cdot n^{3}$ for some positive constant $c$. The regular $n$-gon shows that this bound is tight up to a constant factor. Moreover, we will show that for the corresponding problem for $n$ arbitrary points in $\mathbf{R}^{2}$ one has $\sum_{i=1}^{t} m_{i}^{2} \leq c \cdot n^{3.25}$, where $c$ is a positive constant.

In Section 6 we will give the new lower bound $c \cdot n^{2 / 3}$ ( $c$ a positive constant) on the size of a $B_{2}$-subset of the set $\left\{1^{2}, 2^{2}, \ldots, n^{2}\right\}$. This improves earlier results of Alon and Erdös $[\mathrm{AE}]$.

Finally, in Section 7 we consider some algorithmic aspects of these selection problems under a more general setting. In particular, using derandomization we will give an efficient sequential algorithm that finds in every edge coloring of the complete graph $K_{n}$ a totally multicolored complete subgraph of size at least as large as guaranteed by the probabilistic method. This algorithm has running time $O\left(n^{2} \ln n+\sum_{i} m_{i}^{2}\right)$, where $m_{i}$ is the number of edges in color $i$.

\section{An Anti-Ramsey Result}

In this section we will prove a so-called anti-Ramsey theorem, which we will use for the proofs of Theorems 3 and 5 . Before stating it we will introduce some notation. For further references to anti-Ramsey results we refer to [ALR].

A graph $G$ with vertex set $V$ and edge set $E$ is denoted by $G=(V, E)$. By $K_{n}$ we denote the complete graph on $n$ vertices. A mapping $f: E\left(K_{n}\right) \longrightarrow T$ is called an edge coloring of $K_{n}$ with colors $t \in T$. For $t \in T, f^{-1}(t)$ is the set of all edges colored by color $t$. By $\bar{d}_{t}=\frac{2 \cdot\left|f^{-1}(t)\right|}{n}$ we denote the average degree of color $t \in T$. Let $d_{t}$ be the maximum degree of color $t$, i.e. the maximum number of edges in color $t$, incident at some vertex, and let $\Delta=\max \left\{d_{t} \mid t \in T\right\}$. Finally, a complete subgraph $K_{k}$ of $K_{n}$ is called totally multicolored if the restriction $f \mid E\left(K_{k}\right)$ to the edge set of $K_{k}$ is a one-to-one coloring. 
Theorem 6 For every $\gamma>0$ there exists a constant $C=C(\gamma)>0$, such that for all integers $n \geq 2$ the following holds.

Let $f: E\left(K_{n}\right) \longrightarrow T$ be a coloring and suppose $\tau$ satisfies the following conditions

(i) $\tau \geq \sum_{t \in T} \bar{d}_{t}^{2}$, and

(ii) $\tau \geq n^{1 / 2+\gamma} \cdot \Delta^{3 / 2}$.

Then there exists a totally multicolored subgraph $K_{k}$ of $K_{n}$ with

$$
k \geq C \cdot\left(\frac{n^{2}}{\tau}\right)^{1 / 3} \cdot(\ln n)^{1 / 3} .
$$

For the proof of Theorem 6 we will use the concept of uncrowded hypergraphs. Let $\mathcal{G}=(V, \mathcal{E})$ be a hypergraph with vertex set $V$ and edge set $\mathcal{E}$. For a vertex $v \in V$ let $\operatorname{deg}_{\mathcal{G}}(v)$ denote the degree of $v$ in $\mathcal{G}$, i.e. the number of edges $E \in \mathcal{E}$ containing $v$. By $\operatorname{Deg}(\mathcal{G})=\max \left\{\operatorname{deg}_{\mathcal{G}}(v) \mid v \in V\right\}$ we denote the maximum degree of $\mathcal{G}$. A hypergraph is $k$-uniform if each edge $E \in \mathcal{E}$ has cardinality $k$. A 2-cycle in $\mathcal{G}$ is given by a set of two distinct edges from $\mathcal{E}$, which intersect in at least two elements. A hypergraph is called uncrowded if it contains no 2-cycle. Finally, the independence number $\alpha(\mathcal{G})$ is the maximum cardinality of a subset of $V$ which contains no edges from $\mathcal{E}$.

We will use the following result from [DLR], which is an extension of a theorem from [AKPSS]:

Theorem 7 [DLR] Let $\mathcal{G}=(V, \mathcal{E})$ be a $k$-uniform hypergraph, $k \geq 3$, with $|V|=n$ and maximum degree $\operatorname{Deg}(\mathcal{G}) \leq t^{k-1}$. If

(i) $\mathcal{G}$ contains no 2-cycles, and

(ii) $t$ is sufficiently large, i.e. $t \geq t_{0}(k)$

then

$$
\alpha(\mathcal{G}) \geq c_{k} \cdot \frac{n}{t} \cdot(\ln t)^{\frac{1}{k-1}},
$$

where $c_{k}>0$ is a constant depending only on $k$.

We will now prove Theorem 6 .

Proof: It is sufficient to prove the theorem for sufficiently large $n$, say $n \geq n_{0}$. To see this assume the theorem holds for $n \geq n_{0}$ for some $n_{0}$ and some constant $C>0$. For values of $n$ less than $n_{0}$ the lower bound of the theorem is less than $C n_{0}^{1 / 2}\left(\ln n_{0}\right)^{1 / 3}$. By adapting the constant $C$ the inequality (3) holds for all $n$. Thus we can assume that $n$ is sufficiently large throughout the proof.

Let $V=\{1,2, \ldots, n\}$ be the vertex set of a complete graph $K_{n}$ and let $f: E\left(K_{n}\right) \longrightarrow T$ be an edge coloring. Let $\tau$ satisfy requirements (i) and (ii) in Theorem 6 . We can also assume that $\tau$ satisfies

$$
\tau<n^{2} \ln n,
$$

since otherwise the assertion (3) is trivial and we are done. 
We will construct $i$-uniform hypergraphs $\mathcal{G}_{i}=\left(V, \mathcal{E}_{i}\right), i=3$, 4 , with the same vertex set as follows:

$$
\begin{aligned}
\left\{v_{1}, v_{2}, v_{3}\right\} & \in \mathcal{E}_{3} \\
\left\{v_{1}, v_{2}, v_{3}, v_{4}\right\} & \Leftrightarrow \mathcal{E}_{4} \quad \Leftrightarrow \quad f\left(\left\{v_{1}, v_{2}\right\}\right)=f\left(\left\{v_{1}, v_{3}\right\}\right) \\
& \left.\Leftrightarrow f\left(v_{1}, v_{2}\right\}\right)=f\left(\left\{v_{3}, v_{4}\right\}\right) .
\end{aligned}
$$

Observe that a subset $X \subseteq V$ yields a totally multicolored complete subgraph if and only if $X$ is an independent set in both $\mathcal{G}_{3}$ and $\mathcal{G}_{4}$. Our aim will be to give a lower bound for the maximum size of such an independent set. We cannot apply Theorem 7 directly, as the $\mathcal{G}_{i}, i=3,4$, are in general not uncrowded. To come to such an uncrowded situation we will pick a random subset of the vertex set $V$, and show that an induced subhypergraph can be made uncrowded.

First we will give upper bounds for the cardinalities of $\mathcal{E}_{3}$ and $\mathcal{E}_{4}$. For $\left|\mathcal{E}_{3}\right|$ note that every pair $\{v, w\}$ of vertices can be extended in at most $2 \Delta-2$ ways to an edge $E \in \mathcal{E}_{3}$. Thus,

$$
\left|\mathcal{E}_{3}\right|<\left(\begin{array}{l}
n \\
2
\end{array}\right) \cdot 2 \cdot \Delta<n^{2} \cdot \Delta
$$

Concerning the size of $\mathcal{E}_{4}$ we obviously have

$$
\left|\mathcal{E}_{4}\right| \leq \sum_{t \in T}\left(\begin{array}{c}
\left|f^{-1}(t)\right| \\
2
\end{array}\right) .
$$

As $2 \cdot\left|f^{-1}(t)\right|=\bar{d}_{t} \cdot n$, it follows with (i) that

$$
\left|\mathcal{E}_{4}\right| \leq \sum_{t \in T}\left(\begin{array}{c}
\frac{\bar{d}_{t} \cdot n}{2} \\
2
\end{array}\right)<\frac{n^{2}}{8} \cdot \sum_{t \in T} \bar{d}_{t}^{2} \leq \frac{1}{8} \cdot n^{2} \cdot \tau .
$$

Next we will count the number of 2 -cycles in $\mathcal{G}_{4}$. Let $c_{2}(\mathcal{G})$ denote the number of 2 cycles in a hypergraph $\mathcal{G}=(V, \mathcal{E})$. We will count the 2-cycles more carefully: for $j=2,3$ let $c_{2, j}(\mathcal{G})$ be the number of $(2, j)$-cycles, i.e. the number of pairs $\left\{E, E^{\prime}\right\} \in[\mathcal{E}]^{2}$ with $\left|E \cap E^{\prime}\right|=j$. Clearly, $c_{2}(\mathcal{G})=c_{2,2}(\mathcal{G})+c_{2,3}(\mathcal{G})$.

Concerning $c_{2,2}\left(\mathcal{G}_{4}\right)$, choose an edge $E \in \mathcal{E}_{4}$ and then pick a pair $\{v, w\} \subset E$ of vertices. The number of edges $E^{\prime} \in \mathcal{E}_{4}$ with $E \cap E^{\prime}=\{v, w\}$ is less than the number of pairs $\{x, y\}$ with $f(\{v, w\})=f(\{x, y\})$ or $f(\{v, x\})=f(\{w, y\})$. There are at most $2 n \Delta$ such pairs, hence with $(6)$ we have

$$
c_{2,2}\left(\mathcal{G}_{4}\right) \leq\left|\mathcal{E}_{4}\right| \cdot\left(\begin{array}{l}
4 \\
2
\end{array}\right) \cdot 2 \cdot n \cdot \Delta \leq \frac{3}{2} \cdot n^{3} \cdot \tau \cdot \Delta
$$

To count the number of $(2,3)$-cycles, we fix an edge $E \in \mathcal{E}_{4}$ and a three-element subset $S \subset E$. Then $S$ can be extended in at most $\left(\begin{array}{l}3 \\ 2\end{array}\right) \cdot \Delta$ ways to an edge $E^{\prime} \in \mathcal{E}_{4}$, hence

$$
c_{2,3}\left(\mathcal{G}_{4}\right) \leq\left|\mathcal{E}_{4}\right| \cdot\left(\begin{array}{l}
4 \\
3
\end{array}\right) \cdot\left(\begin{array}{l}
3 \\
2
\end{array}\right) \cdot \Delta \leq \frac{3}{2} \cdot n^{2} \cdot \tau \cdot \Delta
$$

Now we choose a random subset of $V$ by picking each vertex with probability

$$
p=n^{-1 / 3+\epsilon} \cdot \tau^{-1 / 3},
$$


where $0<\epsilon<\gamma / 12$, independently of the other vertices. For the random subset $V^{\prime} \subseteq V$ consider the induced random subhypergraphs $\mathcal{G}_{i}^{\prime}=\left(V^{\prime}, \mathcal{E}_{i}^{\prime}\right)$ for $i=3$, where $\mathcal{E}_{i}^{\prime}=$ $\mathcal{E}_{i} \cap\left[V^{\prime}\right]^{i}$. Moreover, let $c_{2, i}\left(V^{\prime}\right), i=2,3$ be random variables counting the number of $(2, i)$-cycles in $\mathcal{G}_{4}^{\prime}$.

Assumption (4) makes sure that $p n \rightarrow \infty$ as $n \rightarrow \infty$, so we have

$$
\operatorname{Prob}\left(\left|V^{\prime}\right| \approx p \cdot n\right)=1-o(1)
$$

by Chernoff's inequality.

For a random variable $X$ let $E(X)$ denote its expectation. From (5) and (ii) we obtain for $\epsilon<\frac{\gamma}{3}$ that

$$
E\left(\left|\mathcal{E}_{3}^{\prime}\right|\right)=p^{3} \cdot\left|\mathcal{E}_{3}\right|<p^{3} \cdot n^{2} \cdot \Delta=p n \cdot \frac{n^{1 / 3+2 \epsilon} \cdot \Delta}{\tau^{2 / 3}} \leq p n \cdot \frac{1}{n^{2 / 3 \cdot \gamma-2 \epsilon}}=o(p n) .
$$

By (7) and (8) we infer for $\epsilon<\frac{\gamma}{12}$

$$
\begin{aligned}
E\left(c_{2}\left(\mathcal{G}_{4}^{\prime}\right)\right) & =p^{6} \cdot c_{2,2}\left(\mathcal{G}_{4}\right)+p^{5} \cdot c_{2,3}\left(\mathcal{G}_{4}\right) \leq \frac{3}{2} \cdot p^{6} \cdot n^{3} \cdot \tau \cdot \Delta+\frac{3}{2} \cdot p^{5} \cdot n^{2} \cdot \tau \cdot \Delta \\
& =\frac{3 p n}{2} \cdot\left(\frac{n^{1 / 3+5 \epsilon} \cdot \Delta}{\tau^{2 / 3}}+\frac{\Delta}{n^{1 / 3-4 \epsilon} \cdot \tau^{1 / 3}}\right) \\
& \leq \frac{3 p n}{2} \cdot\left(n^{5 \epsilon-2 / 3 \cdot \gamma}+\frac{\Delta^{1 / 2}}{n^{1 / 2+\gamma / 3-4 \epsilon}}\right)=o(p n) .
\end{aligned}
$$

Moreover, we have

$$
E\left(\left|\mathcal{E}_{4}^{\prime}\right|\right)=p^{4} \cdot\left|\mathcal{E}_{4}\right|
$$

Using Markov's inequality, we infer with (9), (10), (11) and (12) that there exists a subset $V^{\prime} \subseteq V$ with $\left|V^{\prime}\right| \approx p \cdot n$, such that the induced hypergraphs $\mathcal{G}_{i}^{\prime}=\left(V^{\prime}, \mathcal{E}_{i}^{\prime}\right), i=3,4$, satisfy the following: $\left|\mathcal{E}_{3}^{\prime}\right|=o(p n)$ and $c_{2}\left(\mathcal{G}_{4}^{\prime}\right)=o(p n)$ and also $\left|\mathcal{E}_{4}^{\prime}\right| \leq 2 \cdot p^{4} \cdot\left|\mathcal{E}_{4}\right|$. Now, delete one vertex from each triple $E \in \mathcal{E}_{3}^{\prime}$ and from each 2-cycle in $\mathcal{G}_{4}^{\prime}$. Moreover, delete all vertices of degree bigger than

$$
\frac{24 \cdot p^{4} \cdot\left|\mathcal{E}_{4}\right|}{p n}=\frac{24 \cdot p^{3} \cdot\left|\mathcal{E}_{4}\right|}{n} .
$$

For $n$ sufficiently large, we obtain a subset $V^{*} \subseteq V^{\prime}$ of at least $\frac{p n}{2}$ vertices containing no edge from $\mathcal{E}_{3}$ and such that the induced subhypergraph $\mathcal{G}_{4}^{*}=\left(V^{*},\left[V^{*}\right]^{4} \cap \mathcal{E}_{4}\right)$ has no 2-cycle and has maximum degree

$$
\operatorname{Deg}\left(\mathcal{G}_{4}^{*}\right) \leq \frac{24 \cdot p^{3} \cdot\left|\mathcal{E}_{4}\right|}{n} \leq 3 p^{3} n \tau=3 n^{3 \epsilon}=t^{3},
$$

by (6), with $t$ defined by the equation. We apply Theorem 7 to the hypergraph $\mathcal{G}_{4}^{*}$ and infer

$$
\alpha\left(\mathcal{G}_{4}^{*}\right) \geq c_{4} \cdot \frac{p n / 2}{3^{1 / 3} \cdot p n^{1 / 3} \tau^{1 / 3}} \cdot\left(\ln \left(3^{1 / 3} \cdot n^{\epsilon}\right)\right)^{1 / 3} \geq C \cdot\left(\frac{n^{2}}{\tau}\right)^{1 / 3} \cdot(\ln n)^{1 / 3} .
$$


Corollary 8 Let $f: E\left(K_{n}\right) \longrightarrow T$ be a coloring of the edges of the complete graph on $n$ vertices, where $\Delta=O\left(n^{1-\beta}\right)$ for a fixed $\beta>0$.

Then, for $n \geq 2$ there exists a totally multicolored subgraph $K_{k}$ with

$$
k \geq c \cdot\left(\frac{n}{\Delta}\right)^{1 / 3} \cdot(\ln n)^{1 / 3}
$$

where $c=c(\beta)>0$ is a constant.

Proof: By Theorem 6 with $\tau=n \cdot \Delta$ and taking $\gamma<\frac{\beta}{2}$.

\section{Two Results from Number Theory}

In this section we are concerned with two results from analytic number theory that we will need for the proofs of Theorem 3 and 5 . For convenience we will use Vinogradov's notation $f(n) \ll g(n)$ for $f(n)=O(g(n))$.

Definition 9 Let $r_{d}(m)$ be the number of representations of $m$ in the form

$$
m=x_{1}^{2}+x_{2}^{2}+\ldots+x_{d}^{2},
$$

where $x_{1}, x_{2}, \ldots, x_{d}$ are integers.

The following result for the 2-dimensional case is due to Ramanujan (see also [Wi]).

\section{Theorem 10 [Rm]}

$$
\sum_{m=1}^{n}\left(r_{2}(m)\right)^{2}=\Theta(n \ln n) .
$$

In fact Ramanujan determines also the leading constant.

Here we will give an alternative proof for the upper bound. Our approach uses simple geometric considerations and might be of interest by itself. For doing so and for later purposes we will use the following definition and lemma.

Definition 11 Let $P$ be a finite set of points in the plane. Consider the bipartite graph $\mathcal{B}=\left([P]^{2} \cup P, I\right)$ with

$$
(\{p, q\}, z) \in I \Longleftrightarrow z \text { lies on the perpendicular bisector of } p \text { and } q \text {. }
$$

Then define $\Delta(P):=|I|$.

Roughly speaking, $\Delta(P)$ is the number of incidences between perpendicular bisectors determined by $P$ and points of $P$ (each bisector can be generated by several pairs of points!). Note that $\Delta(P)$ is nearly the same as the number of isosceles triangles determined by $P$ apart from the fact that equilateral triangles are counted 3 times. 
Lemma 12 Let $P$ be a set of $n$ points in the plane $\mathbf{R}^{2}$. Let the points of $P$ determine distinct distances $d_{1}, d_{2}, \ldots, d_{t}$, where $d_{i}$ occurs with multiplicity $m_{i}$ for $i=1,2, \ldots, t$. Then,

$$
\sum_{i=1}^{t} m_{i}^{2} \leq \frac{n}{2} \cdot\left(\Delta(P)+\left(\begin{array}{l}
n \\
2
\end{array}\right)\right) .
$$

The idea of the proof is similar to an argument of Szemerédi (see [E]).

Proof: Let $P$ be the set of $n$ given points in the plane. Notice that a point $z$ lies on the bisector of $p$ and $q$ if and only if $p$ and $q$ have the same distance from $z$. For $z \in P$ and $i=1,2, \ldots, t$ let $m_{i}(z)$ denote the number of points in $P$, which have distance $d_{i}$ from $z$. Using Jensen's inequality we infer that

$$
\begin{aligned}
\Delta(P) & =\sum_{z \in P} \sum_{i=1}^{t}\left(\begin{array}{c}
m_{i}(z) \\
2
\end{array}\right)=\sum_{i=1}^{t} \sum_{z \in P}\left(\begin{array}{c}
m_{i}(z) \\
2
\end{array}\right) \geq \sum_{i=1}^{t} n \cdot\left(\begin{array}{c}
\frac{2 m_{i}}{n} \\
2
\end{array}\right)=\frac{2}{n} \cdot \sum_{i=1}^{t} m_{i}^{2}-\sum_{i=1}^{t} m_{i} \\
& =\frac{2}{n} \cdot \sum_{i=1}^{t} m_{i}^{2}-\left(\begin{array}{l}
n \\
2
\end{array}\right) .
\end{aligned}
$$

Thus,

$$
\sum_{i=1}^{t} m_{i}^{2} \leq \frac{n}{2} \cdot\left(\Delta(P)+\left(\begin{array}{l}
n \\
2
\end{array}\right)\right) .
$$

Lemma 13 Let $G_{n}$ be the set of points of the $n \times n$-grid $\{0,1, \ldots, n-1\} \times\{0,1, \ldots, n-1\}$. Then,

$$
\Delta\left(G_{n}\right) \leq c \cdot n^{4} \cdot \ln n
$$

for some positive constant c.

Note that $\Delta\left(G_{n}\right)$ is equal to the number of isosceles triangles since $G_{n}$ contains no equilateral triangle.

Proof: For distinct points $p_{1}, p_{2}$ in the $n \times n \operatorname{grid} G_{n}$, where $p_{1}=\left(x_{1}, y_{1}\right)$ and $p_{2}=\left(x_{2}, y_{2}\right)$, let $l$ be the line through $p_{1}$ and $p_{2}$ and define

$$
s(l)=\max \left\{\frac{1}{g} \cdot\left|x_{2}-x_{1}\right|, \frac{1}{g} \cdot\left|y_{2}-y_{1}\right|\right\},
$$

where $g=\operatorname{gcd}\left(x_{2}-x_{1}, y_{2}-y_{1}\right)$. Note that $s(l)$ only depends on $l$ and is independent of the choice of $p_{1}$ and $p_{2}$. We observe that $\left|l \cap G_{n}\right| \leq \frac{n}{s(l)}$. Let $l^{\prime}$ be the perpendicular bisector of $p_{1}$ and $p_{2}$, then we also have $\left|l^{\prime} \cap G_{n}\right| \leq \frac{n}{s(l)}$.

To bound $\Delta\left(G_{n}\right)$ we fix an arbitrary point $p_{1} \in P$ and an integer $1 \leq s \leq n$. Choose a line $l$ through $p_{1}$ with $s(l)=s$. The number of such lines is at most $4 s$. Then we select 
a point $p_{2} \in l \cap G_{n}$ and a point $z \in l^{\prime} \cap G_{n}$, where $l^{\prime}$ is the perpendicular bisector of $p_{1}$ and $p_{2}$. For both $p_{2}$ and $z$ there are at most $\frac{n}{s}$ possibilities. Thus

$$
\Delta\left(G_{n}\right) \leq n^{2} \cdot \sum_{s=1}^{n} 4 s \cdot \frac{n}{s} \cdot \frac{n}{s}=4 n^{4} \cdot \sum_{s=1}^{n} \frac{1}{s} \leq 4 n^{4} \cdot\left(1+\int_{1}^{n} \frac{1}{x} d x\right) \leq c \cdot n^{4} \cdot \ln n .
$$

Corollary 14 Let $G_{n}$ be the set of points of the $n \times n$ grid. For $i=1,2, \ldots, 2(n-1)^{2}$ let $m_{i}$ denote the occurrence of distance $\sqrt{i}$ between different points of $G_{n}$. Then,

$$
\sum_{i=1}^{2(n-1)^{2}} m_{i}^{2} \leq c_{2} \cdot n^{6} \cdot \ln n
$$

for some positive constant $c_{2}$.

Proof: By Lemmas 12 and 13 we obtain

$$
\sum_{i=1}^{2(n-1)^{2}} m_{i}^{2} \leq \frac{n^{2}}{2} \cdot\left(c \cdot n^{4} \cdot \ln n+\left(\begin{array}{c}
n^{2} \\
2
\end{array}\right)\right) \leq c_{2} \cdot n^{6} \cdot \ln n .
$$

Corollary 15 There exists a positive constant $c_{3}$ such that for every positive integer $n$

$$
\sum_{i=1}^{n}\left(r_{2}(i)\right)^{2} \leq c_{3} \cdot n \cdot \ln n
$$

Proof: Consider a circle of radius at most $\frac{n-1}{2}$ around an arbitrary point of the $n \times n$-grid. Then at least a quarter of this circle lies inside the $n \times n$-grid. Thus

$$
\sum_{i=1}^{\left(\frac{n-1}{2}\right)^{2}}\left(n^{2} \cdot \frac{r_{2}(i)}{4}\right)^{2} \leq \sum_{i=1}^{2(n-1)^{2}} m_{i}^{2}
$$

where $m_{i}$ denotes the occurrence of distance $\sqrt{i}$ between different points in the $n \times n$ grid. By Corollary 14 we deduce $\sum_{i=1}^{n}\left(r_{2}(i)\right)^{2} \leq c_{3} \cdot n \cdot \ln n$ for some positive constant $c_{3}$.

Remark. By using the same ideas it is also possible to prove the lower bound in Theorem 10 .

For higher dimensions we will show 


\section{Theorem 16}

$$
\sum_{m=1}^{n}\left(r_{d}(m)\right)^{2}=O\left(n^{d-1}\right), \quad \text { for } d \geq 3
$$

Remark. It can be shown that the bound given in Theorem 16 is asymptotically sharp by using for example Cauchy's inequality together with the fact that $\sum_{m=1}^{n} r_{d}(m) \approx$ $\frac{\pi^{d / 2}}{\Gamma(d / 2+1)} n^{d / 2}$.

We are going to prove Theorem 16 by using the Hardy-Littlewood circle-method (see [Dp], [Vg]). Throughout the remaining part of this section let $N:=\lfloor\sqrt{n}\rfloor$ and suppose $d \geq 3$. Define $R_{d}(m, N)$ as the number of representations of $m$ in the form

$$
m=x_{1}^{2}+x_{2}^{2}+\ldots+x_{d}^{2}, \quad 1 \leq x_{i} \leq N .
$$

Lemma 17 Let $\epsilon>0$ and $N=\lfloor\sqrt{n}\rfloor$, then

$$
\sum_{m=1}^{n}\left(r_{d}(m)\right)^{2} \leq c_{d} \sum_{m=1}^{n}\left(R_{d}(m, N)\right)^{2}+O\left(n^{d-3 / 2+\epsilon}\right)
$$

with a constant $c_{d}$ depending only on $d$.

Proof: It is easy to see that we can estimate

$$
R_{d}(m, N) \leq r_{d}(m) \leq 2^{d} \cdot R_{d}(m, N)+d \cdot r_{d-1}(m)
$$

for $m \leq n$. As $r_{2}(m)=O\left(m^{\epsilon^{\prime}}\right)$ for every $\epsilon^{\prime}>0$, we have

$$
r_{s}(m) \leq(\sqrt{m})^{s-2} \cdot O\left(m^{\epsilon^{\prime}}\right)=O\left(m^{s / 2-1+\epsilon^{\prime}}\right)
$$

for $s \geq 2$. We can then estimate

$$
\left(r_{d}(m)\right)^{2} \leq 2^{2 d} \cdot\left(R_{d}(m, N)\right)^{2}+O\left(m^{d-5 / 2+\epsilon}\right)
$$

and the assertion follows.

Define $e(y):=e^{2 \pi i y}$ and $f(\alpha):=\sum_{x=1}^{N} e\left(\alpha x^{2}\right)$ for real numbers $\alpha$.

\section{Lemma 18}

$$
\sum_{m=1}^{d n}\left(R_{d}(m, N)\right)^{2}=\int_{0}^{1}|f(\alpha)|^{2 d} d \alpha .
$$

Proof: First we observe that

$$
(f(\alpha))^{d}=\sum_{x_{1}=1}^{N} \ldots \sum_{x_{d}=1}^{N} e\left(\alpha \cdot\left(x_{1}^{2}+\ldots+x_{d}^{2}\right)\right)=\sum_{m=1}^{d n} R_{d}(m, N) \cdot e(\alpha m),
$$


hence,

$$
\left|(f(\alpha))^{d}\right|^{2}=\sum_{m_{1}=1}^{d n} R_{d}\left(m_{1}, N\right) \cdot e\left(\alpha m_{1}\right) \sum_{m_{2}=1}^{d n} R_{d}\left(m_{2}, N\right) \cdot e\left(-\alpha m_{2}\right) .
$$

If we integrate this we obtain

$$
\int_{0}^{1}|f(\alpha)|^{2 d} d \alpha=\sum_{1 \leq m_{1}, m_{2} \leq d n} R_{d}\left(m_{1}, N\right) \cdot R_{d}\left(m_{2}, N\right) \cdot \int_{0}^{1} e\left(\alpha \cdot\left(m_{1}-m_{2}\right)\right) d \alpha .
$$

The integral on the right hand side is 0 for $m_{1} \neq m_{2}$ and 1 for $m_{1}=m_{2}$, thus

$$
\int_{0}^{1}|f(\alpha)|^{2 d} d \alpha=\sum_{m=1}^{d n}\left(R_{d}(m, N)\right)^{2}
$$

In order to estimate $\int_{0}^{1}|f(\alpha)|^{2 d} d \alpha$ we break the integration interval into two parts called major-arcs and minor-arcs. The major-arcs will determine the order of magnitude while the contribution of the minor-arcs will be negligible.

In the following calculations let $0<\delta<1 / 5$ be a fixed real number. For integers $a, q$ with $1 \leq a \leq q \leq N^{\delta}$ and $(a, q)=1$ define the major-arcs as

$$
\mathcal{M}_{a, q}:=\left\{\alpha \in \mathbf{R}:|\alpha-a / q|<N^{-2+\delta}\right\} .
$$

We observe that these major-arcs are disjoint since their lengths are much smaller than the distances between their centers. Let $\mathcal{M}$ denote the union of the $\mathcal{M}_{a, q}$. It is convenient to shift the integration interval $(0,1]$ to the right to $U:=\left(N^{-2+\delta}, 1+N^{-2+\delta}\right]$. As $f(\alpha)=$ $f(\alpha+1)$ we have

$$
\int_{0}^{1}|f(\alpha)|^{2 d} d \alpha=\int_{U}|f(\alpha)|^{2 d} d \alpha .
$$

Now $\mathcal{M} \subset U$ by definition. The set $\mathrm{M}:=U \backslash \mathcal{M}$ forms the minor-arcs.

To see that the minor-arcs can be neglected in order to prove Theorem 16 we use the following

Lemma 19 Suppose $s \geq 5$, then

$$
\int_{\mathrm{M}}|f(\alpha)|^{s} d \alpha=O\left(n^{s / 2-1-\delta^{\prime}}\right),
$$

where $\delta^{\prime}$ is a constant depending on $\delta$.

This lemma can be found for example in [Dp] and [Vg]. Using this, we immediately get for $d \geq 3$, that

$$
\int_{M}|f(\alpha)|^{2 d} d \alpha=O\left(n^{d-1-\delta^{\prime}}\right)
$$


where $\delta^{\prime}$ is a constant depending on $\delta$.

Thus it remains to prove that

$$
\int_{\mathcal{M}}|f(\alpha)|^{2 d} d \alpha=O\left(n^{d-1}\right) .
$$

Define

$$
S_{a, q}:=\sum_{z=1}^{q} e\left(a z^{2} / q\right) \quad \text { and } \quad I(\beta):=\int_{0}^{N} e\left(\beta \xi^{2}\right) d \xi .
$$

For the treatment of the major-arcs we use the following lemmas.

Lemma 20 [Dp] If $\alpha \in \mathcal{M}_{a, q}$, then

$$
f(\alpha)=V(\alpha)+O\left(N^{2 \delta}\right)
$$

with $V(\alpha):=q^{-1} \cdot S_{a, q} \cdot I(\alpha-a / q)$.

The notation $V(\alpha)$ is a bit sloppy because $V$ also depends on $a$ and $q$. Nevertheless there should be no confusion since $\alpha$ determines the major-arc $\mathcal{M}_{a, q}$ in which it lies.

Lemma 21 [Dp] Let $a, q$ be relative prime integers with $q>0$. Then for every $\epsilon>0$,

$$
\left|S_{a, q}\right|=O\left(q^{1 / 2+\epsilon}\right) \text {. }
$$

Now let $\alpha \in \mathcal{M}_{a, q}$. Lemma 20 and the obvious fact that

$$
|V(\alpha)|=\left|q^{-1} \cdot S_{a, q} \cdot I(\alpha-a / q)\right| \leq N
$$

yields

$$
(f(\alpha))^{2 d}=(V(\alpha))^{2 d}+O\left(N^{2 d-1+2 \delta}\right) .
$$

Since the measure of $\mathcal{M}$ is bounded by $O\left(N^{-2+3 \delta}\right)$ and since $\delta<1 / 5$ we can again neglect the $O$-term and consider only $\int_{\mathcal{M}}|V(\alpha)|^{2 d} d \alpha$.

By Lemma 21 we see that

$$
|V(\alpha)|^{2 d} \leq\left|q^{-1} S_{a, q}\right|^{2 d} \cdot|I(\alpha-a / q)|^{2 d} \ll q^{-d+\epsilon^{\prime}} \cdot|I(\alpha-a / q)|^{2 d}
$$

for $\alpha \in \mathcal{M}_{a, q}$. Substituting $\alpha=a / q+\beta$ implies

$$
\int_{\mathcal{M}_{a, q}}|V(\alpha)|^{2 d} d \alpha \ll q^{-d+\epsilon^{\prime}} \cdot \int_{|\beta|<N^{-2+\delta}}|I(\beta)|^{2 d} d \beta
$$

Summing up over all major-arcs gives

$$
\sum_{q=1}^{N^{\delta}} \sum_{\substack{a=1 \\(a, q)=1}}^{q} \int_{\mathcal{M}_{a, q}}|V(\alpha)|^{2 d} d \alpha \ll \sum_{q=1}^{N^{\delta}} \sum_{\substack{a=1 \\(a, q)=1}}^{q} q^{-d+\epsilon^{\prime}} \cdot \int_{|\beta|<N^{-2+\delta}}|I(\beta)|^{2 d} d \beta
$$




$$
\leq\left(\sum_{q=1}^{\infty} q^{1-d+\epsilon^{\prime}}\right) \cdot \int_{|\beta|<N^{-2+\delta}}|I(\beta)|^{2 d} d \beta=\text { const. } \int_{|\beta|<N^{-2+\delta}}|I(\beta)|^{2 d} d \beta .
$$

The infinite sum converges since $d \geq 3$. By substituting $\xi=N \cdot t$ and $\beta=N^{-2} \cdot \gamma$ in $I(\beta)=\int_{0}^{N} e\left(\beta \xi^{2}\right) d \xi$ we obtain

$$
\begin{aligned}
\int_{|\beta|<N^{-2+\delta}}|I(\beta)|^{2 d} d \beta & =N^{2 d-2} \cdot \int_{|\gamma|<N^{\delta}}\left|\int_{0}^{1} e\left(\gamma t^{2}\right) d t\right|^{2 d} d \gamma \\
& \leq n^{d-1} \cdot \int_{|\gamma|<N^{\delta}}\left|\int_{0}^{1} e\left(\gamma t^{2}\right) d t\right|^{2 d} d \gamma,
\end{aligned}
$$

since $N=\lfloor\sqrt{n}\rfloor$.

We want to show that the outer integral is bounded from above independent of $n$. By substituting $t=\gamma^{1 / 2} z$ and by the fact that $\int_{0}^{\infty} \cos \left(x^{2}\right) d x$ and $\int_{0}^{\infty} \sin \left(x^{2}\right) d x$ are bounded one can show that

$$
\left|\int_{0}^{1} e\left(\gamma t^{2}\right) d t\right|=\left|\gamma^{-1 / 2} \cdot \int_{0}^{\gamma^{1 / 2}} e\left(z^{2}\right) d z\right| \ll \gamma^{-1 / 2},
$$

for $\gamma \geq 0$. On the other hand, obviously

$$
\left|\int_{0}^{1} e\left(\gamma t^{2}\right) d t\right| \leq 1
$$

This enables us to extend the integration in (15) to infinity:

$$
\begin{aligned}
\int_{-\infty}^{+\infty}\left|\int_{0}^{1} e\left(\gamma t^{2}\right) d t\right|^{2 d} d \gamma & =\int_{-1}^{+1}\left|\int_{0}^{1} e\left(\gamma t^{2}\right) d t\right|^{2 d} d \gamma+2 \cdot \int_{1}^{+\infty}\left|\int_{0}^{1} e\left(\gamma t^{2}\right) d t\right|^{2 d} d \gamma \\
& \ll 2 \cdot 1+2 \cdot \int_{1}^{+\infty} \gamma^{-d} d \gamma=O(1)
\end{aligned}
$$

since $d \geq 3$. There we made use of (16) and (17). Thus we have proved

Lemma 22

$$
\int_{\mathcal{M}}|f(\alpha)|^{2 d} d \alpha=O\left(n^{d-1}\right) .
$$

Because of the fact that $\int_{0}^{1}=\int_{\mathcal{M}}+\int_{M}$ and by (14) we have

\section{Corollary 23}

$$
\int_{0}^{1}|f(\alpha)|^{2 d} d \alpha=O\left(n^{d-1}\right) .
$$

Now Lemma 17 and 18 and Corollary 23 imply Theorem 16. 


\section{Grid Points}

In this section we will give the proofs for Theorems 3 and 5 . First we will show Theorem 3.

Proof: Given the $n \times n$-grid $G_{n}$, we form a complete graph $K_{n^{2}}$ with vertex set $\{1,2, \ldots, n\} \times$ $\{1,2, \ldots, n\}$. We color the edges $\{x, y\}$ by the square of the euclidean distance of their endpoints $x$ and $y$. For fixed positive integer $t$ and every grid point $v$, notice that the number of grid points $w$ with euclidean distance $\sqrt{t}$ from $v$ is bounded from above by the number of representations of $t$ as a sum of two squares. Hence, the average degrees $\bar{d}_{t}$ of color $t$ satisfy $\bar{d}_{t} \leq r_{2}(t)$, and for the maximum degree $\Delta$ we have that

$$
\Delta \leq n^{c_{1} / \ln \ln n},
$$

where $c_{1}$ is a positive constant, by a result of Wigert, $c f .[H W]$. By Theorem 10 we have

$$
\sum_{t=1}^{2(n-1)^{2}} \bar{d}_{t}^{2} \leq \sum_{t=1}^{2(n-1)^{2}}\left(r_{2}(t)\right)^{2}=c_{2} \cdot n^{2} \cdot \ln n
$$

where $c_{2}$ is a positive constant. Setting $\tau=C \cdot n^{2} \cdot \ln n$ for a positive constant $C$, which is large enough, we see with (18) that (i) and (ii) in Theorem 6 are satisfied (notice that the number of vertices is $n^{2}$ ). Hence, there exists a totally multicolored complete subgraph on $k$ vertices with

$$
k \geq c^{\prime} \cdot\left(\frac{n^{4}}{n^{2} \cdot \ln n}\right)^{1 / 3} \cdot\left(\ln n^{2}\right)^{1 / 3} \geq c \cdot n^{2 / 3} .
$$

The vertices of this subgraph determine $k$ points in the $n \times n$-grid with mutual distinct distances. We remark that we could have also used Corollary 14 to obtain the same conclusion.

Next we will prove Theorem 5:

Proof: Fix a positive integer $d \geq 3$. We proceed as in the proof given above by coloring the edges of the complete graph on $n^{d}$ vertices by the square of the euclidean distances of the corresponding endpoints. Clearly, $\bar{d}_{t} \leq r_{d}(t)$ and

$$
\Delta \leq n^{d / 2-1+\epsilon^{\prime}}
$$

for any fixed $\epsilon^{\prime}>0$ by (13).

Now,

$$
\sum_{t=1}^{d(n-1)^{2}} \bar{d}_{t}^{2} \leq \sum_{t=1}^{d(n-1)^{2}}\left(r_{d}(t)\right)^{2}=O\left(d^{d-1} \cdot n^{2 d-2}\right)
$$

by Theorem 16 . With $\tau=C \cdot n^{2 d-2}$, where $C>0$ is a large enough constant, requirements (i) and (ii) of Theorem 6 are satisfied. Hence there exists a totally multicolored complete subgraph on $k$ vertices with

$$
k \geq c(d) \cdot\left(\frac{n^{2 d}}{n^{2 d-2}}\right)^{1 / 3} \cdot\left(\ln n^{d}\right)^{1 / 3} \geq c_{d} \cdot n^{2 / 3} \cdot(\ln n)^{1 / 3},
$$


which yields the desired result.

\section{Points in Arbitrary Position}

In this section we will study the maximum cardinality of a subset of $n$ arbitrary points in the euclidean plane $\mathbf{R}^{2}$, such that the mutual distances among the points of $X$ are distinct. Moreover, we will consider the same question for $n$ points in general position (no three on a line) in $\mathbf{R}^{2}$.

Theorem 24 Let $n$ arbitrary points in the plane $\mathbf{R}^{2}$ be given. Let the $n$ points determine distinct distances $d_{1}, d_{2}, \ldots, d_{t}$, where distance $d_{i}$ occurs with multiplicity $m_{i}$ for $i=1,2, \ldots, t$.

Then

$$
\sum_{i=1}^{t} m_{i}^{2} \leq c \cdot n^{13 / 4}
$$

where $c>0$ is a constant.

Remarks. (1) Spencer, Szemerédi and Trotter proved in [SST] that under the assumptions of Theorem 24 one has $m_{i} \leq c^{\prime} \cdot n^{4 / 3}$ for $i=1,2, \ldots, t$, where $c^{\prime}$ is a positive constant. Their result applied in the straightforward way yields $\sum_{i=1}^{t} m_{i}^{2} \leq \max \left\{m_{1}, m_{2}, \ldots, m_{t}\right\}$. $\sum_{i=1}^{t} m_{i} \leq c^{\prime} \cdot n^{4 / 3} \cdot\left(\begin{array}{l}n \\ 2\end{array}\right) \leq c n^{10 / 3}$ for a positive constant $c$. Another way to get this upper bound is by using the result of Pach and Sharir [PS] that the number of isosceles triangles is bounded by $O\left(n^{7 / 3}\right)$. Then by Lemma 12 one obtains that $\sum_{i=1}^{t} m_{i}^{2} \leq c_{2} n^{10 / 3}$, where $c_{2}$ is a positive constant.

(2) For the points of the $\sqrt{n} \times \sqrt{n}$-grid we have $\sum m_{i}^{2}=\Theta\left(n^{3} \ln n\right)$. One might conjecture that this upper bound holds for any set of $n$ points in the euclidean plane.

For the proof of Theorem 24 we will use

Lemma 25 Let $0=S_{0} \leq S_{1} \leq \ldots \leq S_{t}$ and $m_{1} \geq m_{2} \geq \ldots \geq m_{t} \geq 0$ be sequences of real numbers such that

$$
\sum_{i=1}^{j} m_{i} \leq S_{j} \quad \text { for } j=1,2, \ldots, t .
$$

Then

$$
\sum_{i=1}^{t} m_{i}^{2} \leq \sum_{i=1}^{t}\left(S_{i}-S_{i-1}\right)^{2} .
$$

Proof: We will apply induction on $t$. For $t=1$ the assertion is trivial, hence assume $t>1$ and that the conclusion of the lemma holds for all values $1,2, \ldots, t-1$. For given $S_{0}, S_{1}, \ldots, S_{t}$ it suffices to show $(21)$ for any sequence $m_{1} \geq m_{2} \geq \ldots \geq m_{t}$ satisfying (20) and maximizing the expression $\sum_{i=1}^{t} m_{i}^{2}$. Let $m_{1}, m_{2}, \ldots, m_{t}$ be such a sequence. If 
$m_{t}=0$, then $(21)$ is clearly satisfied by the induction hypothesis, hence we can assume that $m_{t} \neq 0$.

Suppose first that $\sum_{i=1}^{j} m_{i}<S_{j}$ for all $j=1,2, \ldots, t-1$. Then define a new sequence $m_{1}^{*}, m_{2}^{*}, \ldots, m_{t}^{*}$ as follows

$$
\begin{aligned}
& m_{1}^{*}=m_{1}+\epsilon \\
& m_{i}^{*}=m_{i} \quad \text { for } i=2,3, \ldots, t-1 \\
& m_{t}^{*}=m_{t}-\epsilon
\end{aligned}
$$

where $\epsilon=\min \left\{m_{t}, S_{1}-m_{1}, S_{2}-\left(m_{1}+m_{2}\right), \ldots, S_{t-1}-\sum_{i=1}^{t-1} m_{i}\right\}$. By assumption $\epsilon>$ 0 , and hence $\sum_{i=1}^{t} m_{i}^{* 2}>\sum_{i=1}^{t} m_{i}^{2}$, which contradicts the maximality of the sequence $m_{1}, m_{2}, \ldots, m_{t}$.

Thus there is a $k, 1 \leq k \leq t-1$, with

$$
\sum_{i=1}^{k} m_{i}=S_{k}
$$

By the induction assumption it follows that

$$
\sum_{i=1}^{k} m_{i}^{2} \leq \sum_{i=1}^{k}\left(S_{i}-S_{i-1}\right)^{2}
$$

By (20) and (22) we have $\sum_{i=k+1}^{j} m_{i} \leq S_{j}-S_{k}$ for $j=k+1, k+2, \ldots, t$. Using again the induction assumption we infer that

$$
\sum_{i=k+1}^{t} m_{i}^{2} \leq \sum_{i=k+1}^{t}\left(\left(S_{i}-S_{k}\right)-\left(S_{i-1}-S_{k}\right)\right)^{2}=\sum_{i=k+1}^{t}\left(S_{i}-S_{i-1}\right)^{2}
$$

thus combining (23) and (24) we obtain $\sum_{i=1}^{t} m_{i}^{2} \leq \sum_{i=1}^{t}\left(S_{i}-S_{i-1}\right)^{2}$, which finishes the induction step.

Let $P$ be a set of points in the plane $\mathbf{R}^{2}$ and let $C$ be a set of circles in $\mathbf{R}^{2}$. Define a bipartite graph $G$ with vertex set $P \cup C$ and edge set $E$, where $(p, c) \in E, p \in P$ and $c \in C$, if and only if $p$ lies on the circle $c$. Let $I(P, C)$ denote the number of incidences between points and circle, that is, the number of edges in this bipartite graph $G$. In our arguments we will use the following result of Clarkson, Edelsbrunner, Guibas, Sharir and Welzl:

Theorem 26 [CEGSW] Let $P$ be a set of points in $\mathbf{R}^{2}$ and let $C$ be a set of circles in $\mathbf{R}^{2}$. Then

$$
I(P, C)=O\left(|P|^{3 / 5} \cdot|C|^{4 / 5}+|P|+|C|\right) .
$$

Now we are ready to prove Theorem 24: 
Proof: Let $P \subset \mathbf{R}^{2}$ be a set of $n$ points in the plane. Assume that $m_{1} \geq m_{2} \geq \ldots \geq m_{t}$. Around each point $p \in P$ draw circles with radius $d_{1}, d_{2}, \ldots, d_{t}$. For $j=1,2, \ldots, t$ let $C_{j}$ be the set of all such circles with radius $d_{1}, d_{2}, \ldots, d_{j}$. Then we have $\left|C_{j}\right|=j n$ and

$$
I\left(P, C_{j}\right)=2 \cdot \sum_{i=1}^{j} m_{i}
$$

Thus, by (25) we have

$$
I\left(P, C_{j}\right) \leq c_{1} \cdot n^{3 / 5} \cdot(j n)^{4 / 5},
$$

where $c_{1}>0$ is a constant.

Combining (26) and (27), we infer that

$$
\sum_{i=1}^{j} m_{i} \leq \frac{c_{1}}{2} \cdot n^{7 / 5} \cdot j^{4 / 5}
$$

for $j=1,2, \ldots, t$.

On the other hand, we have

$$
\sum_{i=1}^{l} m_{i} \leq\left(\begin{array}{l}
n \\
2
\end{array}\right)<\frac{n^{2}}{2}
$$

for $l=1,2, \ldots, t$.

Put $c_{2}=\max \left\{1 / 2, c_{1} / 2\right\}$ and

$$
S_{j}= \begin{cases}c_{2} \cdot n^{7 / 5} \cdot j^{4 / 5} & \text { if } 0 \leq j \leq n^{3 / 4} \\ c_{2} \cdot n^{2} & \text { if } n^{3 / 4}<j \leq t .\end{cases}
$$

Clearly, the sequences $S_{0}, S_{1}, \ldots, S_{t}$ and $m_{1}, m_{2}, \ldots, m_{t}$ satisfy the assumptions of Lemma 25. Hence,

$$
\sum_{i=1}^{t} m_{i}^{2} \leq \sum_{i=1}^{t}\left(S_{i}-S_{i-1}\right)^{2} \leq \sum_{i=1}^{n^{3 / 4}}\left(S_{i}-S_{i-1}\right)^{2} \leq c_{2}^{2} \cdot n^{14 / 5} \cdot \sum_{i=1}^{n^{3 / 4}}\left(i^{4 / 5}-(i-1)^{4 / 5}\right)^{2} .
$$

Since $i^{4 / 5}-(i-1)^{4 / 5} \leq i^{-1 / 5}$ for $i \geq 1,(29)$ becomes

$$
\sum_{i=1}^{t} m_{i}^{2} \leq c_{2}^{2} \cdot n^{14 / 5} \cdot \sum_{i=1}^{n^{3 / 4}} i^{-2 / 5}
$$

The function $g(x)=x^{-2 / 5}, x>0$, is decreasing, thus

$$
\sum_{i=1}^{n^{3 / 4}} i^{-2 / 5} \leq 1^{-2 / 5}+\int_{1}^{n^{3 / 4}} x^{-2 / 5} d x=1+\frac{5}{3}\left(n^{9 / 20}-1\right)<\frac{5}{3} n^{9 / 20} .
$$

We infer with (30) that

$$
\sum_{i=1}^{t} m_{i}^{2} \leq c \cdot n^{13 / 4}
$$


for some constant $c>0$.

Surprisingly, the situation changes radically if our point set is in general position, i.e. no three points lie on a common line. More generally, we have

Theorem 27 Let $P$ be a set of $n$ points in the plane $\mathbf{R}^{2}$ such that at most s points are on a line. If these points determine distinct distances $d_{1}, d_{2}, \ldots, d_{t}$ with corresponding multiplicities $m_{1}, m_{2}, \ldots, m_{t}$, then

$$
\sum_{i=1}^{t} m_{i}^{2} \leq \frac{(s+1) n}{2} \cdot\left(\begin{array}{l}
n \\
2
\end{array}\right) .
$$

Remark. If one assumes in contrast to Theorem 27 that the $n$ points lie on $s$ lines (instead of 'at most $s$ points on a line'), then one can show by a similar argument that $\sum m_{i}^{2} \leq s n\left(\begin{array}{l}n \\ 2\end{array}\right)$ holds.

Proof: We will give an upper bound for $\Delta(P)$ (see Definition 11). By assumption the perpendicular bisector of any two points $p$ and $q$ contains at most $s$ points of $P$. Thus,

$$
\Delta(P) \leq s \cdot\left(\begin{array}{l}
n \\
2
\end{array}\right)
$$

By Lemma 12 we infer that

$$
\sum_{i=1}^{t} m_{i}^{2} \leq \frac{n}{2} \cdot\left(\Delta(P)+\left(\begin{array}{l}
n \\
2
\end{array}\right)\right) \leq \frac{(s+1) n}{2} \cdot\left(\begin{array}{l}
n \\
2
\end{array}\right) .
$$

Theorem 28 Let $P$ be a set of $n$ points in the plane $\mathbf{R}^{2}$ in general position. If these points determine the distinct distances $d_{1}, d_{2}, \ldots, d_{t}$ with multiplicities $m_{1}, m_{2}, \ldots, m_{t}$, then

$$
\sum_{i=1}^{t} m_{i}^{2} \leq \frac{3}{4} n^{2}(n-1) .
$$

If we further assume that the $n$ points are in convex position, then

$$
\sum_{i=1}^{t} m_{i}^{2} \leq \frac{3}{4} n^{2}(n-1)-\frac{n^{2}}{2} .
$$

This proves a conjecture of Erdös and Fishburn [Fi], [EF]. In their paper [EF] an even stronger statement is conjectured namely that for convex $n$-gons one has

$$
\text { i } \quad \sum_{i=1}^{t} m_{i}^{2} \leq \frac{n^{2} \cdot(n-1)}{2} \quad ?
$$


for $n \geq 3$ being an odd integer. For $n \geq 10$ an even integer it is stated in [EF] that perhaps

$$
\text { ¿ } \quad \sum_{i=1}^{t} m_{i}^{2} \leq \frac{n^{2} \cdot(2 n-3)}{4} \quad \text { ? }
$$

In both cases the regular convex $n$-gon would be an extremal configuration attaining the upper bounds. For $n=4,6,8$ they proved that this bound does not hold.

Remarks. (1) Füredi proved in [Fü] that under the assumptions of Theorem 28, i.e., $P$ is a convex $n$-gon, one has $m_{i} \leq 12 n \log n$ for $i=1,2, \ldots, t$. Applying this in the straightforward way, one gets $\sum_{i=1}^{t} m_{i}^{2} \leq \sum_{i=1}^{t} m_{i} \cdot \max \left\{m_{1}, m_{2}, \ldots, m_{t}\right\} \leq 6 n^{3} \cdot \log n$.

(2) According to the second remark after Theorem 24 we see, that it really makes a difference to assume general position.

Proof: If the points of $P$ are in general position, then (33) follows by Theorem 27 with $s=2$. So assume that the points of $P$ determine a convex $n$-gon. Observe that the bisector of $x$ and $y$ contains at most one point from $P$ if $x$ and $y$ are adjacent along the boundary of the convex hull of $P$. Thus,

$$
\Delta(P) \leq 2\left(\begin{array}{l}
n \\
2
\end{array}\right)-n
$$

By Lemma 12 we infer that

$$
\sum_{i=1}^{t} m_{i}^{2} \leq \frac{n}{2}\left(2\left(\begin{array}{l}
n \\
2
\end{array}\right)-n+\left(\begin{array}{l}
n \\
2
\end{array}\right)\right)=\frac{3 n^{2}(n-1)}{4}-\frac{n^{2}}{2} .
$$

It might be worth noting that the following upper bounds for the sum $\sum_{i=1}^{t} m_{i}^{2}$ match the conjectured upper bounds of Erdös and Fishburn stated above.

Theorem 29 Let $P$ be a set of $n$ points in $\mathbf{R}^{2}$, which has the following property: $\left.{ }^{*}\right) \quad$ no circle with center $p \in P$ contains three or more other points of $P$.

Let these $n$ points determine distinct distances $d_{1}, d_{2}, \ldots, d_{t}$ with corresponding multiplicities $m_{1}, m_{2}, \ldots, m_{t}$. Then,

$$
\sum_{i=1}^{t} m_{i}^{2} \leq \begin{cases}\frac{n^{2}(n-1)}{2} & \text { if } n \text { is odd } \\ \frac{n^{2}(2 n-3)}{4} & \text { if } n \text { is even. }\end{cases}
$$

Proof: By $(*)$ we have $m_{i} \leq n$ for $i=1,2, \ldots, t$. Thus

$$
\sum_{i=1}^{t} m_{i}^{2} \leq \frac{\left(\begin{array}{l}
n \\
2
\end{array}\right)}{n} n^{2}=\frac{n^{2}(n-1)}{2},
$$

which shows the assertion for $n$ being an odd integer.

For $n$ even, consider as above the bipartite graph $G=\left([P]^{2} \cup P, E\right)$ with $\{\{x, y\}, z\} \in$ $E$ if and only if $z$ lies on the perpendicular bisector of $x$ and $y$. To determine $|E|=\Delta(P)$, 
fix a point $p \in P$. Let $d(p)$ denote the degree of $p$ in $G$. Then $d(p)$ is equal to the number of unordered pairs $\{x, y\} \in[P \backslash\{p\}]^{2}$ such that $p$ lies on the perpendicular bisector of $x$ and $y$. Property $(*)$ implies that these pairs form a matching. As $n$ is even, we obtain $d(p) \leq(n-2) / 2$ and therefore

$$
\Delta(P)=\sum_{p \in P} d(p) \leq \frac{n(n-2)}{2} .
$$

With Lemma 12 we infer that

$$
\sum_{i=1}^{t} m_{i}^{2} \leq \frac{n}{2}\left(\Delta(P)+\left(\begin{array}{l}
n \\
2
\end{array}\right)\right) \leq \frac{n^{2}(2 n-3)}{4} .
$$

Next we will consider the corresponding selection problems.

Theorem 30 Let $P$ be a set of $n$ points in general position in the plane. Then there exists a subset $X \subseteq P$ with mutual distinct distances such that

$$
|X| \geq c \cdot n^{1 / 3}
$$

for some positive constant $c>0$.

Remark. An upper bound of $O\left(n^{1 / 2}\right)$ is given by the regular $n$-gon.

Proof: Let $P=\left\{p_{1}, p_{2}, \ldots, p_{n}\right\}$ be given as above. Let $d_{1}, d_{2}, \ldots, d_{t}$ be the occurring distinct distances with corresponding multiplicities $m_{1}, m_{2}, \ldots, m_{t}$. We will construct a hypergraph $\mathcal{H}=\left(P, \mathcal{E}_{3} \cup \mathcal{E}_{4}\right)$ as follows.

Let $\left\{p_{i}, p_{j}, p_{k}\right\} \in \mathcal{E}_{3} \subseteq[P]^{3}$ if and only if $d\left(p_{i}, p_{j}\right)=d\left(p_{i}, p_{k}\right)$, where $d(p, q)$ denotes the euclidean distance between $p$ and $q$. Moreover, $\left\{p_{i}, p_{j}, p_{k}, p_{l}\right\} \in \mathcal{E}_{4} \subseteq[P]^{4}$ if and only if $d\left(p_{i}, p_{j}\right)=d\left(p_{k}, p_{l}\right)$.

Consider two points $p, p^{\prime} \in P$ and let $Q=\left\{q \in P: d(q, p)=d\left(q, p^{\prime}\right)\right\}$. We observe that all points in $Q$ lie on the perpendicular bisector of $p$ and $p^{\prime}$. Since $P$ is in general position we know that $|Q| \leq 2$, thus

$$
\left|\mathcal{E}_{3}\right| \leq\left(\begin{array}{l}
n \\
2
\end{array}\right) \cdot 2<n^{2}
$$

Concerning $\left|\mathcal{E}_{4}\right|$, we have by Theorem 24 that

$$
\left|\mathcal{E}_{4}\right| \leq \sum_{i=1}^{t}\left(\begin{array}{c}
m_{i} \\
2
\end{array}\right) \leq c_{1} \cdot n^{3} .
$$

We make a random experiment consisting of two steps. First choose a random subset $X \subset P$ by selecting points independently with probability $p=c_{2} \cdot n^{-2 / 3}$, where $c_{2}>0$ is a constant that will be specified later. In the second step we delete from $X$ one point from each edge in $\mathcal{E}_{3}^{*}=[X]^{3} \cap \mathcal{E}_{3}$ and $\mathcal{E}_{4}^{*}=[X]^{4} \cap \mathcal{E}_{4}$. By (34) and (35) this results in an independent set $Y \subset X$ with average size

$$
\begin{aligned}
E(|Y|) & \geq E(|X|)-E\left(\left|\mathcal{E}_{3}^{*}\right|\right)-E\left(\left|\mathcal{E}_{4}^{*}\right|\right)=p \cdot n-p^{3} \cdot\left|\mathcal{E}_{3}\right|-p^{4} \cdot\left|\mathcal{E}_{4}\right| \\
& >c_{2} n^{1 / 3}-c_{2}^{3}-c_{1} c_{2}^{4} n^{1 / 3} .
\end{aligned}
$$


Choosing $c_{2}=\min \left\{3^{-1 / 2},\left(3 c_{1}\right)^{-1 / 3}\right\}$, we obtain that

$$
E(|Y|)>\frac{c_{2}}{3} \cdot n^{1 / 3}
$$

Hence there exists a set $Y \subset P$ of the desired size and with distinct mutual distances.

Using Theorem 27 one can show using (31) and (32) in a similar fashion the following Theorem 31 Let $P$ be set of $n$ points in the plane $\mathbf{R}^{2}$ such that at most $s$ points of $P$ lie on any line. Then there exists a subset $X \subseteq P$ with mutual distinct distances such that

$$
|X| \geq c \cdot\left(\frac{n}{s}\right)^{1 / 3}
$$

where $c>0$ is an absolute constant (independent of $s$ ).

For $n$ points in arbitrary position we have the following result.

Theorem 32 Let $P$ be a set of $n$ points in $\mathbf{R}^{2}$. Then there exists a subset $X \subseteq P$ with mutual distinct distances such that

$$
|X| \geq c \cdot n^{1 / 4}
$$

where $c>0$ is a constant.

This improves a former result from [Th], where the lower bound $|X| \geq c \cdot n^{2 / 9}$ has been given. An upper bound of $O\left(n^{1 / 2} /(\log n)^{1 / 4}\right)$ follows from the $\sqrt{n} \times \sqrt{n}$-grid.

Proof: The arguments are similar to those used in the proof of Theorem 30 . We form a hypergraph $\mathcal{H}=\left(P, \mathcal{E}_{3} \cup \mathcal{E}_{4}\right)$ as before. Pach and Sharir have shown in [PS] that $n$ points in the plane determine $O\left(n^{7 / 3}\right)$ isosceles triangles, hence

$$
\left|\mathcal{E}_{3}\right| \leq c_{1} \cdot n^{7 / 3}
$$

and by Theorem 24

$$
\left|\mathcal{E}_{4}\right| \leq c_{2} \cdot n^{13 / 4} .
$$

By choosing vertices at random with probability $p=c_{3} \cdot n^{-3 / 4}$ for some small enough positive constant $c_{3}$ we obtain as above a subset $Y \subseteq P$ with mutual distinct distances such that $|Y| \geq c \cdot n^{1 / 4}$.

With Theorem 24 we see that for an $n$-point set $P$ in $\mathbf{R}^{2}$ the fraction of those $k$-element subsets of $P$ which determine less than $\left(\begin{array}{l}k \\ 2\end{array}\right)$ distinct distances is bounded from above by

$$
\frac{\sum_{i=1}^{t} m_{i}^{2} \cdot\left(\begin{array}{l}
n-4 \\
k-4
\end{array}\right)+O\left(n^{7 / 3}\right) \cdot\left(\begin{array}{l}
n-3 \\
k-3
\end{array}\right)}{\left(\begin{array}{l}
n \\
k
\end{array}\right)} \leq O\left(\frac{k^{4}}{n^{3 / 4}}+\frac{k^{3}}{n^{2 / 3}}\right)
$$

Thus if $k=o\left(n^{3 / 16}\right)$ then almost all $k$-element subsets of $P$ determine distinct mutual distances. This improves former results from [AEP] and [Th], where $k=o\left(n^{1 / 7}\right)$ respective $k=o\left(n^{1 / 6}\right)$ has been shown. In [AEP] it was stated with respect to an upper bound for this problem that for $n$ equidistant points on a line and supposing $k=\Omega\left(n^{1 / 4}\right)$, then a positive percentage of all $k$-sets determine less than $\left(\begin{array}{l}k \\ 2\end{array}\right)$ distinct distances. Here we will make this statement more precise in the following form: 
Theorem 33 Let $p_{1}, p_{2}, \ldots, p_{n}$ be $n$ equidistant points on a line. Then the number of $k$-element subsets of $\left\{p_{1}, p_{2} \ldots, p_{n}\right\}$, which determine less than $\left(\begin{array}{l}k \\ 2\end{array}\right)$ distinct distances, is at least

$$
\left(1-\frac{c}{k}-\frac{c n}{k^{4}}\right) \cdot\left(\begin{array}{l}
n \\
k
\end{array}\right)
$$

where $c$ is a positive constant.

Proof: Let $P=\left\{p_{1}, p_{2}, \ldots, p_{n}\right\}$ be the set of equidistant points on a line. Form a hypergraph $\mathcal{G}=\left(P, \mathcal{E}_{3} \cup \mathcal{E}_{4}\right)$ with $\mathcal{E}_{3} \subseteq[P]^{3}$ and $\mathcal{E}_{4} \subseteq[P]^{4}$ as follows: $\left\{p_{i}, p_{j}, p_{k}\right\} \in \mathcal{E}_{3}$ if and only if $d\left(p_{i}, p_{j}\right)=d\left(p_{i}, p_{k}\right)$. Moreover, let $\left\{p_{i}, p_{j}, p_{k}, p_{l}\right\} \in \mathcal{E}_{4}$ if and only if $d\left(p_{i}, p_{j}\right)=$ $d\left(p_{k}, p_{l}\right)$ (where $d$ denotes the euclidean distance). Clearly,

$$
c_{4} n^{3} \leq\left|\mathcal{E}_{4}\right| \leq c_{4}^{\prime} n^{3} .
$$

Let $\mathcal{E}_{4}=\left\{S_{1}, S_{2}, \ldots, S_{t}\right\}$, where $c_{4} \leq \frac{t}{n^{3}} \leq c_{4}^{\prime}$. Now pick a $k$-element subset $K$ uniformly at random among the set of all $k$-element subsets of $\mathcal{V}$. Let $E$ denote the event that $[K]^{3} \cap \mathcal{E}_{3}=\emptyset$ and $[K]^{4} \cap \mathcal{E}_{4}=\emptyset$. In the following we will determine an upper bound for the probability that $E$ occurs. For $i=1,2, \ldots, t$ let $z_{i}$ be indicator random variables for the events $S_{i} \subseteq K$, i.e.

$$
z_{i}= \begin{cases}1 & \text { if } S_{i} \subseteq K \\ 0 & \text { else. }\end{cases}
$$

Define another random variable $Z=\sum_{i=1}^{t} z_{i}$ and let $E(Z)$ be its expected value. Then by Chebychev's inequality

$$
\operatorname{Prob}(E) \leq \operatorname{Prob}(Z=0) \leq \frac{\operatorname{Var}(Z)}{E(Z)^{2}}
$$

By linearity of expectation we have

$$
E(Z)=\sum_{i=1}^{t} E\left(z_{i}\right)=t \cdot \frac{\left(\begin{array}{l}
n-4 \\
k-4
\end{array}\right)}{\left(\begin{array}{l}
n \\
k
\end{array}\right)}=t \cdot \frac{[k]_{4}}{[n]_{4}},
$$

where $[n]_{l}=n \cdot(n-1) \cdot \ldots \cdot(n-l+1)$ denotes the falling factorial.

For $s=5,6,7,8$ let $a_{s}$ denote the number of unordered pairs $\left\{S_{i}, S_{j}\right\}, 1 \leq i<j \leq t$, with $\left|S_{i} \cup S_{j}\right|=s$. Then we have for the variance that

$$
\begin{aligned}
\operatorname{Var}(Z) & =E\left((Z-E(Z))^{2}\right) \\
& =2 \cdot \sum_{1 \leq i<j \leq t} E\left(z_{i} z_{j}\right)+E(Z)-E(Z)^{2} \\
& =2 \cdot \sum_{s=5}^{8} a_{s} \cdot \frac{[k]_{s}}{[n]_{s}}+E(Z)-E(Z)^{2}
\end{aligned}
$$

Next we will give upper bounds on $a_{s}, 5 \leq s \leq 8$. First fix an edge $E \in \mathcal{E}_{4}$. For the following considerations notice that for a fixed distance $d>0$ and every integer $i$, $1 \leq i \leq n$, the number of points $p_{j}, 1 \leq j \leq n$, with $d\left(p_{i}, p_{j}\right)=d$ is at most two. 
To bound $a_{5}$, choose a three-element subset $R \subset E$. Then $R$ can be extended to an edge in $\mathcal{E}_{4} \backslash\{E\}$ in at most 6 ways, hence

$$
a_{5} \leq 24 t
$$

To bound $a_{6}$, choose a two-element subset $R$ of $E$. Then $R$ can be extended in at most $2(n-4)$ ways to an edge in $\mathcal{E}_{4} \backslash\{E\}$, hence

$$
a_{6} \leq t \cdot\left(\begin{array}{l}
4 \\
2
\end{array}\right) \cdot 2 \cdot(n-4) \leq 12 n t .
$$

To bound $a_{7}$, take an element $x \in E$. Then the number of edges $E^{*} \in \mathcal{E}_{4} \backslash\{E\}$ with $x \in E^{*}$ is at most $(n-4) \cdot(n-5) \cdot 2$, thus

$$
a_{7} \leq 8 n^{2} t
$$

Finally, we have

$$
a_{8} \leq\left(\begin{array}{l}
t \\
2
\end{array}\right)
$$

Inserting (40), (41), (42) and (43) in (39) yields for $k, n \geq 7$

$$
\begin{aligned}
\operatorname{Var}(Z) & \leq 48 t \cdot \frac{[k]_{5}}{[n]_{5}}+24 t n \cdot \frac{[k]_{6}}{[n]_{6}}+16 t n^{2} \cdot \frac{[k]_{7}}{[n]_{7}}+2 \cdot\left(\begin{array}{l}
t \\
2
\end{array}\right) \cdot \frac{[k]_{8}}{[n]_{8}}+t \cdot \frac{[k]_{4}}{[n]_{4}}-\left(t \cdot \frac{[k]_{4}}{[n]_{4}}\right)^{2} \\
& \leq 88 t n^{2} \cdot \frac{[k]_{7}}{[n]_{7}}+t \cdot \frac{[k]_{4}}{[n]_{4}}-t \cdot \frac{[k]_{8}}{[n]_{8}} \leq 88 t n^{2} \cdot \frac{[k]_{7}}{[n]_{7}}+t \cdot \frac{[k]_{4}}{[n]_{4}} .
\end{aligned}
$$

With (36), (37), (38) and (44) we infer for $k, n \geq 7$ that

$$
\begin{aligned}
\operatorname{Prob}(E) & \leq \operatorname{Prob}(Z=0) \leq \frac{t \cdot\left(88 n^{2} \cdot \frac{[k]_{7}}{[n]_{7}}+\frac{[k]_{4}}{[n]_{4}}\right)}{t^{2} \cdot\left(\frac{[k]_{4}}{[n]_{4}}\right)^{2}} \\
& \leq 88 \cdot \frac{n^{3}}{t \cdot k}+\frac{[n]_{4}}{[k]_{4} \cdot t} \leq \frac{88}{k \cdot c_{4}}+\frac{81 \cdot n}{k^{4} \cdot c_{4}} \leq \frac{c}{k}+\frac{c \cdot n}{k^{4}},
\end{aligned}
$$

where $c=\frac{88}{c_{4}}$.

Corollary 34 For $k=\omega\left(n^{1 / 4}\right)$ and $n$ equidistant points on a line almost all $k$-element subsets determine less than $\left(\begin{array}{l}k \\ 2\end{array}\right)$ distinct distances.

Considerations, similar to those in the proof of Theorem 33 yield the following

Corollary 35 Let $p_{1}, p_{2}, \ldots, p_{n}$ be the points of the regular n-gon. Then the number of $k$-element subsets of $\left\{p_{1}, p_{2}, \ldots, p_{n}\right\}$, which determine less than $\left(\begin{array}{l}k \\ 2\end{array}\right)$ distinct distances is at least

$$
\left(1-\frac{c}{k}-\frac{c n}{k^{4}}\right) \cdot\left(\begin{array}{l}
n \\
k
\end{array}\right)
$$

for a positive constant $c$. 
By Theorem 24 and (34) it follows that for every $n$-point set $P$ in $\mathbf{R}^{2}$ in general position the fraction of those $k$-element subsets of $P$ which determine less than $\left(\begin{array}{l}k \\ 2\end{array}\right)$ distinct distances is bounded from above by

$$
\frac{\sum_{i=1}^{t} m_{i}^{2} \cdot\left(\begin{array}{l}
n-4 \\
k-4
\end{array}\right)+n^{2} \cdot\left(\begin{array}{l}
n-3 \\
k-3
\end{array}\right)}{\left(\begin{array}{l}
n \\
k
\end{array}\right)} \leq O\left(\frac{k^{4}}{n}+\frac{k^{3}}{n}\right)=O\left(\frac{k^{4}}{n}\right) .
$$

Thus, for $k=o\left(n^{1 / 4}\right)$ almost all $k$-element subsets of $P$ determine distinct mutual distances. By Corollary 35 this bound is tight since almost all $k$-sets of the points of the regular $n$-gon determine less than $\left(\begin{array}{c}k \\ 2\end{array}\right)$ distinct distances for $k=\omega\left(n^{1 / 4}\right)$.

Similar conclusions can be obtained for the $n \times n$-grid $G_{n}$. Namely, construct as in the proof of Theorem 33 a hypergraph $\mathcal{H}=\left(G_{n}, \mathcal{E}_{3} \cup \mathcal{E}_{4}\right)$ with vertex set being the points of the $n \times n$-grid. Then by Theorem 10 we infer

$$
\begin{aligned}
& \left|\mathcal{E}_{3}\right| \leq c_{3} \cdot n^{4} \cdot \ln n \\
& \left|\mathcal{E}_{4}\right| \leq c_{4} \cdot n^{6} \cdot \ln n
\end{aligned}
$$

Thus, by (45) and (46) the fraction of those $k$-element subsets of the $n \times n$-grid $G_{n}$ with less than $\left(\begin{array}{l}k \\ 2\end{array}\right)$ distinct distances is bounded from above by

$$
\frac{\left|\mathcal{E}_{3}\right| \cdot\left(\begin{array}{c}
n^{2}-3 \\
k-3
\end{array}\right)+\left|\mathcal{E}_{4}\right| \cdot\left(\begin{array}{c}
n^{2}-4 \\
k-4
\end{array}\right)}{\left(\begin{array}{c}
n^{2} \\
k
\end{array}\right)} \leq c_{3} \cdot \frac{k^{3} \cdot \ln n}{n^{2}}+c_{4} \cdot \frac{k^{4} \cdot \ln n}{n^{2}} .
$$

Hence, for $k=o\left(\frac{n^{1 / 2}}{(\ln n)^{1 / 4}}\right)$ almost all $k$-element subsets of $G_{n}$ determine distinct mutual distances.

On the other hand, using the ideas of the proof of Theorem 33 with corresponding random variable $Z$ for the $n \times n$-grid and using Theorem 10 and (18) one can show by Chebychev's inequality, that

$$
\operatorname{Prob}(Z=0) \leq \frac{c \cdot n \frac{c^{*}}{\ln \ln n}}{k \cdot \ln n}+\frac{c \cdot n^{2}}{k^{4} \cdot \ln n},
$$

where $c, c^{*}$ are positive constants. Thus for $k=\omega\left(\frac{n^{1 / 2}}{(\ln n)^{1 / 4}}\right)$ almost all $k$-element subsets of the $n \times n$-grid determine less than $\left(\begin{array}{l}k \\ 2\end{array}\right)$ distinct distances.

We remark that one can show that for the corresponding problem for the $n^{d}$-grid, $d \geq 3$, we also have a $0-1$ law with threshold function $f(n)=\sqrt{n}$, as can be seen along the lines above using (13), Theorem 16 and the remark after Theorem 16. In particular, for $k=o\left(n^{1 / 2}\right)$ almost all $k$-element subsets of the $n^{d}$-grid determine distinct mutual distances, while for $k=\omega\left(n^{1 / 2}\right)$ almost all $k$-element subsets of the $n^{d}$-grid determine less than $\left(\begin{array}{l}k \\ 2\end{array}\right)$ distinct distances.

\section{$6 \quad \mathrm{~B}_{2}$-Sets}

For finite sets $X \subset \mathrm{N}$ a subset $S \subseteq X$ is called a $B_{2}$-set (or Sidon set) if all pairwise sums $s+s^{\prime}, s \neq s^{\prime}$, are distinct. One is interested in the maximum size of $S$. For the case $X=\{1,2, \ldots, n\}$ the maximum size of a $B_{2}$-set $S \subseteq X$ is asymptotically well 
known by results from Erdös and Turan to be $\left(\frac{1}{2}+o(1)\right) \cdot n^{1 / 2}$. In [AE] Alon and Erdös considered the maximum size of $B_{2}$-subsets of the set $\left\{1^{2}, 2^{2}, \ldots, n^{2}\right\}$ consisting of the first $n$ squares. Using an idea similar to the one given in the proof of Theorem 30 they showed the following:

Theorem 36 [AE] For every $\epsilon>0$ there exists $c=c(\epsilon)>0$ such that for every positive integer $n$ there exists a $B_{2}$-set $S \subset\left\{1^{2}, 2^{2}, \ldots, n^{2}\right\}$ with

$$
|S| \geq c \cdot n^{2 / 3-\epsilon} \text {. }
$$

As already observed in [AE], by a theorem of Landau [La] one has the upper bound $|S| \leq c^{\prime} \cdot \frac{n}{(\ln n)^{1 / 4}}$. Here we will improve inequality (47), namely we will show:

Theorem 37 For every integer $n \geq 1$ there exists a $B_{2}$-set $S \subset\left\{1^{2}, 2^{2}, \ldots, n^{2}\right\}$ with

$$
|S| \geq c \cdot n^{2 / 3}
$$

where $c>0$ is a constant.

The first idea to prove Theorem 37 might be to consider a complete graph with vertex set $V=\left\{1^{2}, 2^{2}, \ldots, n^{2}\right\}$ and a coloring of the edges, where the edge $\left\{i^{2}, j^{2}\right\}$ receives color $i^{2}+j^{2}$. Then a totally multicolored complete subgraph on $k$ vertices gives rise to a $B_{2^{-}}$ subset of $V$ of cardinality $k$. But Theorem 6 is not applicable to prove Theorem 37, as by condition (ii) we can only guarantee a totally multicolored complete subgraph of size less than $c \cdot n^{1 / 2}$. But it turns out, that with more refined counting arguments a similar strategy as used for the proof of Theorem 6 will show (48):

Proof: As in the proof of Theorem 6 we can assume that $n$ is sufficiently large. In the following $c_{1}, c_{2}, \ldots, c_{10}$ are positive constants. We construct a 4 -uniform hypergraph $\mathcal{G}=(V, \mathcal{E})$ with vertex set $V=\left\{1^{2}, 2^{2}, \ldots, n^{2}\right\}$ and edges $\left\{i^{2}, j^{2}, k^{2}, l^{2}\right\} \in \mathcal{E} \subseteq[V]^{4}$ if and only if $i^{2}+j^{2}=k^{2}+l^{2}$. As the number of representations of any positive integer $x$ by a sum of two squares is given by $r_{2}(x)$, we have by Theorem 10 that

$$
|\mathcal{E}| \leq \sum_{i=1}^{2 n^{2}}\left(\begin{array}{c}
r_{2}(i) \\
2
\end{array}\right) \leq c_{1} \cdot n^{2} \cdot \ln n .
$$

Next we will count the number of 2 -cycles in $\mathcal{G}$. To count $c_{2,2}(\mathcal{G})$ choose an edge $E \in \mathcal{E}$, say $E=\left\{i^{2}, j^{2}, k^{2}, l^{2}\right\}$ where $i^{2}+j^{2}=k^{2}+l^{2}$. There are six possibilities to choose a two-element subset of $E$, say we choose $\left\{i^{2}, j^{2}\right\}$. Then the number of pairs $\left\{x^{2}, y^{2}\right\}$ with $i^{2}+j^{2}=x^{2}+y^{2}$ is bounded from above by $r_{2}\left(i^{2}+j^{2}\right) \leq n^{\frac{c_{2}}{\ln n}}$ ( $\mathrm{cf}$. [HW]). Now consider those pairs $\left\{x^{2}, y^{2}\right\}$ with $i^{2}+x^{2}=j^{2}+y^{2}$. Assuming $j>i$, we have $j^{2}-i^{2}=(x+y) \cdot(x-y)$, i.e. $(x+y)$ divides $j^{2}-i^{2}$. Fixing this divisor fixes both $x$ and $y$. Hence the number of such pairs $\left\{x^{2}, y^{2}\right\}$ is bounded from above by the number of divisors of $\left(j^{2}-i^{2}\right)$, which is at most $n \frac{c_{3}}{\ln \ln n}$ (see [HW]). Summarizing these considerations, we have

$$
c_{2,2}(\mathcal{G}) \leq c_{4} \cdot n^{2} \cdot \ln n \cdot n^{\frac{c_{5}}{\ln \ln n}} .
$$

Concerning $c_{2,3}(\mathcal{G})$, we choose an edge $E \in \mathcal{E}$ and a three-element subset $T \subset E$. Then $T$ can be extended in at most two ways to an edge $E^{\prime} \in \mathcal{E} \backslash\{E\}$, thus

$$
c_{2,3}(\mathcal{G}) \leq c_{6} \cdot n^{2} \cdot \ln n
$$


As in the proof of Theorem 6 we choose a random subset of $V$ by picking vertices $v \in V$, independently of the others, with probability

$$
p=n^{-1 / 3+\epsilon} \cdot(\ln n)^{-1 / 3},
$$

where $\epsilon<\frac{1}{18}$. Let $R$ be the arising random subset of $V$. Then,

$$
\operatorname{Prob}(|R| \approx p n)=1-o(1)
$$

and by (49) we have

$$
E\left(\left|[R]^{4} \cap \mathcal{E}\right|\right)=p^{4} \cdot|\mathcal{E}| \leq c_{1} \cdot \frac{n^{2 / 3+4 \epsilon}}{(\ln n)^{1 / 3}}
$$

The expected number $E\left(c_{2}(R)\right)$ of 2-cycles in the subhypergraph induced on $R$ can be bounded from above by $(50)$ and (51) as follows:

$$
E\left(c_{2}(R)\right)=p^{6} \cdot c_{2,2}(\mathcal{G})+p^{5} \cdot c_{2,3}(\mathcal{G}) \leq c_{4} \cdot \frac{n^{6 \epsilon+\frac{c_{5}}{\ln \ln n}}}{\ln n}+c_{6} \cdot \frac{n^{1 / 3+5 \epsilon}}{(\ln n)^{2 / 3}}=o(p n)
$$

for $\epsilon<\frac{1}{18}$.

As in the proof of Theorem 6 we infer with (52), (53) and (54) by using Chernoff's and Markov's inequality, deleting one point from each 2-cycle and deleting points of degree bigger than, say, twice the average degree, that there exists a subset $Y \subset V,|Y| \geq c_{7} \cdot p \cdot n$ such that the subhypergraph $\mathcal{G}^{\prime}$ of $\mathcal{G}$ induced on $Y$ has no 2-cycles, has at most $c_{8} \cdot p^{4} \cdot|\mathcal{E}|$ edges and has maximum degree at most $t^{3}=c_{9} \cdot n^{3 \epsilon}$. By Theorem 7 applied to $\mathcal{G}^{\prime}$ we see that

$$
\alpha(\mathcal{G}) \geq \alpha\left(\mathcal{G}^{\prime}\right) \geq c_{10} \cdot \frac{n^{2 / 3+\epsilon}}{t \cdot(\ln n)^{1 / 3}} \cdot(\ln t)^{1 / 3} \geq c \cdot n^{2 / 3}
$$

which finishes the proof.

\section{Algorithmic Aspects}

In this section we will discuss some algorithmic aspects of the selection problems considered in this article. All these selection problems can be formulated in terms of edge colorings of complete graphs. The general question is: given an edge coloring $f$ of the complete graph $K_{n}$, what is the maximum size $r(f)$ of a totally multicolored complete subgraph, i.e. a set of vertices determining mutually distinct edge colors. Clearly, this problem is NP-hard. With an edge coloring we associate a hypergraph $\mathcal{H}=\left(V\left(K_{n}\right), \mathcal{E}_{3} \cup \mathcal{E}_{4}\right)$, where $\mathcal{E}_{3}$ is the family of 3 -sets of vertices determining two equal edge colors and $\mathcal{E}_{4}$ is the family of 4 -sets of vertices that determine two equal colors but do not contain a 3 -set from $\mathcal{E}_{3}$. Hence we have $r(f)=\alpha(\mathcal{H})$, where $\alpha(\mathcal{H})$ is the independence number of $\mathcal{H}$.

We define the probabilistic bound

$$
\tilde{\alpha}(\mathcal{H}):=\max _{p \in[0,1]}\left(p n-p^{3}\left|\mathcal{E}_{3}\right|-p^{4}\left|\mathcal{E}_{4}\right|\right),
$$


which is a lower bound for $\alpha(\mathcal{H})$, since we can pick each vertex independently with probability $p$ and then delete one vertex from each edge occurring in the resulting subhypergraph. This gives an independent set of size at least $p n-p^{3}\left|\mathcal{E}_{3}\right|-p^{4}\left|\mathcal{E}_{4}\right|$ in the average. By using derandomization techniques (see [AS]) we can turn this probabilistic argument into a deterministic algorithm that computes an independent set of the hypergraph and thus a totally multicolored complete subgraph of the original graph of size at least $\tilde{\alpha}(\mathcal{H})$. In the following, we will describe the algorithm.

Let the vertex set of $K_{n}$ be $V=\{1,2, \ldots, n\}$. Let $f: E\left(K_{n}\right) \longrightarrow T$ be an edge coloring and assume that $T$ is totally ordered. For $t \in T$ let $m_{t}=\left|f^{-1}(t)\right|$ be the number of edges in color $t$. In a preprocessing we form our hypergraph $\mathcal{H}=\left(V, \mathcal{E}_{3} \cup \mathcal{E}_{4}\right)$ by collecting pairs of edges of the same color. By first sorting the set of edges with respect to their colors this can be done in time $O\left(n^{2} \ln n+\sum_{t \in T} m_{t}^{2}\right)$. Moreover, we use the following data structure. There is a list of the vertices $v \in V$ and a list of the edges $e \in \mathcal{E}_{3} \cup \mathcal{E}_{4}$. For each vertex $v \in V$ there are pointers to all edges containing $v$. For each edge there are pointers to all vertices contained in it.

Knowing $\left|\mathcal{E}_{3}\right|$ and $\left|\mathcal{E}_{4}\right|$, we can easily compute that value $p \in[0,1]$, which maximizes the expression $p n-p^{3}\left|\mathcal{E}_{3}\right|-p^{4}\left|\mathcal{E}_{4}\right|$. Fix this value of $p$.

In the following we will examine the vertices of $V$ one by one and decide whether each vertex belongs to our independent set or not.

Set $\mathcal{E}=\mathcal{E}_{3} \cup \mathcal{E}_{4}$. Suppose that we already made a partial selection of vertices and let $\epsilon_{1}, \epsilon_{2}, \ldots, \epsilon_{j}$ be the 0,1 -sequence representing this selection, that is, for some vertices we determined whether they do $\left(\epsilon_{i}=1\right)$ or do not $\left(\epsilon_{i}=0\right)$ belong to our independent set. Define weight functions $f_{j}$ and $F_{j}$ depending on $\epsilon_{1}, \epsilon_{2}, \ldots, \epsilon_{j}$ as follows. For vertices $v \in V$ let

$$
f_{j}(v)= \begin{cases}\epsilon_{v} & \text { if } v \leq j \\ p & \text { if } v>j\end{cases}
$$

For edges $e \in \mathcal{E}$ set

$$
f_{j}(e)=\prod_{v \in e} f_{j}(v)
$$

Finally, set

$$
F_{j}=F_{j}\left(\epsilon_{1}, \epsilon_{2}, \ldots, \epsilon_{j-1}\right)=\sum_{v \in V} f_{j}(v)-\sum_{\epsilon \in \mathcal{E}} f_{j}(\epsilon) .
$$

Observe that $F_{j}$ is the expected value of the number of vertices minus the number of edges in a random extension of the selection $\epsilon_{1}, \epsilon_{2}, \ldots, \epsilon_{j}$.

At the beginning, for $j=0$, we have $f_{0}(v)=p$ and $f_{0}(e)=p^{|e|}$ for $v \in V$ and $e \in \mathcal{E}$, thus $F_{0}=\tilde{\alpha}(\mathcal{H})$. We will construct a 0 , 1-sequence $\epsilon_{1}, \epsilon_{2}, \ldots, \epsilon_{n}$ such that the values $F_{j}=F_{j}\left(\epsilon_{1}, \ldots, \epsilon_{j}\right)$ are nondecreasing for $j=0,1, \ldots, n$. In particular, we will have $F_{n} \geq F_{0}$.

Now assume that $\epsilon_{1}, \epsilon_{2}, \ldots, \epsilon_{j-1}$ and $F_{j-1} \geq F_{j-2} \geq \ldots \geq F_{0}$ are given. To determine $\epsilon_{j}$ and thus $F_{j}$, we compute the two values

$$
\begin{aligned}
& W_{j}^{0}=F_{j}\left(\epsilon_{1}, \epsilon_{2}, \ldots, \epsilon_{j-1}, 0\right) \\
& W_{j}^{1}=F_{j}\left(\epsilon_{1}, \epsilon_{2}, \ldots, \epsilon_{j-1}, 1\right) .
\end{aligned}
$$

This can be done in time $O\left(1+\operatorname{deg}_{\mathcal{H}}(j)\right)$. If $W_{j}^{0} \geq W_{j}^{1}$, then we set $\epsilon_{j}=0$ and $F_{j}=W_{j}^{0}$. Otherwise, if $W_{j}^{1}>W_{j}^{0}$, then set $\epsilon_{j}=1$ and $F_{j}=W_{j}^{1}$. 
By straightforward calculations or by interpreting $F_{j}$ as an expected value, we derive

$$
F_{j-1}=(1-p) \cdot W_{j}^{0}+p \cdot W_{j}^{1} .
$$

This implies $F_{j} \geq F_{j-1}$.

Continuing in this way, we obtain a 0,1 -sequence $\epsilon_{1}, \epsilon_{2}, \ldots, \epsilon_{n}$ with $F_{n} \geq F_{0}$. Set $I=\left\{v \in V \mid \epsilon_{v}=1\right\}$. We claim that $I$ is an independent set in $\mathcal{H}$. Assume this is not the case. Thus there is an edge $e \in \mathcal{E}$ contained in $I$. Let $j$ be the last vertex of $e$ chosen by our algorithm. Since $j$ was chosen, we know that $W_{j}^{1}>W_{j}^{0}$, which with (55) implies that

$$
F_{j}=W_{j}^{1}>F_{j-1} .
$$

On the other hand, since $j$ is the last chosen vertex of $e$, we infer that

$$
F_{j}-F_{j-1} \leq\left(f_{j}(j)-f_{j-1}(j)\right)-\left(f_{j}(e)-f_{j-1}(e)\right)=(1-p)-(1-p)=0,
$$

a contradiction. Thus $I$ is an independent set with

$$
|I|=F_{n} \geq F_{0}=\tilde{\alpha}(\mathcal{H})
$$

as desired.

Without the preprocessing this algorithm has a linear running time of $O\left(n+\left|\mathcal{E}_{3}\right|+\left|\mathcal{E}_{4}\right|\right)$. Since $\left|\mathcal{E}_{3}\right|,\left|\mathcal{E}_{4}\right|<\sum_{t \in T} m_{t}^{2}$, we have an overall running time of $O\left(n^{2} \ln n+\sum_{t \in T} m_{t}^{2}\right)$.

By our former results we obtain the following typical consequences. By Theorem 28, given a set $P$ of $n$ points in $\mathbf{R}^{2}$ in general position, this algorithm finds in sequential time $O\left(n^{3}\right)$ a subset $X \subseteq P$ with mutual distinct distances of size at least $c \cdot n^{1 / 3}$, where $c$ is a positive constant. Moreover, by Theorem 24, if the $n$ points of $P$ are in arbitrary position, then the algorithm finds in time $O\left(n^{13 / 4}\right)$ a subset $X \subseteq P$ with mutual distinct distances of size $c \cdot n^{1 / 4}$ for some positive constant $c$.

On the other hand, we remark that by the method described by Alon, Babai and Itai [ABI] there is an NC-algorithm that computes a totally multicolored complete subgraph of size at least $c \cdot \tilde{\alpha}(\mathcal{H})$ for some constant $c>0$.

Remark. This research was partly motivated by related problems considered in [EGRT] which have applications to the problem of distance measuring by using radar or sonar signals, see [Go] and [GT].

Acknowledgement. We would like to thank Kent D. Boklan for drawing our attention to the Hardy-Littlewood method. We also thank Emo Welzl for suggesting Lemma 25.

\section{References}

[ABI] N. Alon, L. Babai and A. Itai, A Fast and Simple Randomized Parallel Algorithm for the Maximal Independent Set Problem, Journal of Algorithms 7 (1986), $567-583$.

[AKPSS] M. Ajtai, J. Komlós, J. Pintz, J. Spencer and E. Szemerédi, Extremal Uncrowded Hypergraphs, Journal of Combinatorial Theory Ser. A 32 (1982), 321-335. 
[AE] N. Alon And P. Erdős, An Application of Graph Theory to Additive Number Theory, European Journal of Combinatorics 6 (1980), 201-203.

[ALR] N. Alon, H. Lefmann and V. Rödl, On an Anti-Ramsey Type Result, Colloquia Mathematica Societatis János Bolyai, 60. Sets, Graphs and Numbers, Budapest (1991), 9-22.

[AS] N. Alon and J. Spencer, The Probabilistic Method, Wiley 8 Sons, New York (1992).

[AEP] D. Avis, P. Erdös and J. Pach, Distinct Distances Determined by Subsets of a Point Set in Space, Computational Geometry 1 (1991), 1-11.

[CEgSW] K. Clarkson, H. Edelsbrunner, L. Guibas, M. Sharir and E. Welzi, Combinatorial Complexity Bounds for Arrangements of Curves and Spheres, Discrete and Computational Geometry 5 (1990), 99-160.

[Dp] H. Davenport, Analytic Methods for Diophantine Equations and Diophantine Inequalities, Campus Publishers (1962), 1-30.

[DLR] R. A. Duke, H. Lefmann and V. RödL, On Uncrowded Hypergraphs, preprint, (1993).

[E] P. Erdős, Some Combinatorial and Metric Problems in Geometry, Intuitive Geometry, Coll. Math. Soc. János Bolyai 48 (K. Böröczky and G. Fejes Tóth, eds.), North-Holland, Amsterdam-New York (1987), 167-177.

[EF] P. Erdös and P. C. Fishburn, Multiplicities of Interpoint Distances in Finite Planar Sets, preprint, (1991).

[EG] P. Erdös And R. GuY, Distinct Distances between Lattice Points, Elemente der Mathematik 25 (1970), 121-123.

[EGRT] P. Erdös, R. L. Graham, I. Rusza and H. Taylor, Bounds for Arrays of Dots with Distinct Slopes or Lengths, Combinatorica 12 (1992), 39-44.

[Fi] P. Fish вunn, Convex Polygons with Few Vertices, Dimacs Technical Report 92-17 (1992).

[Fï] Z. Füredi, The Maximum Number of Unit Distances in a Convex $n$-gon, Journal of Combinatorial Theory, Ser. A 55 (1990), 316-320.

[Go] S. W. Golom B, Construction of Signals with Favourable Correlation Properties, Surveys in Combinatorics London Mathematical Society Lecture Note Series 166 (1991), 1-39.

[GT] S. W. Golom and H. Taylor, Two-dimensional Synchronization Patterns for Minimum Ambiguity, IEEE Transactions Information Theory IT-28 (1982), 600-604.

[Gu] R. K. Guy, Unsolved Problems in Number Theory, Springer Verlag, New York (1981), 132-133. 
[HW] G. H. Hardy, E. M. Wright, An Introduction to the Theory of Numbers, Oxford University Press (1979).

[La] E. Landau, Handbuch der Lehre von der Verteilung der Primzahlen, Teubner Verlag, Leipzig (1909).

[PS] J. PACh And M. Sharir, Repeated Angles in the Plane and Related Problems, Journal of Combinatorial Theory Ser. A 59 (1992), 12-22.

[Rm] S. Ramanujan, Collected Papers, Chelsea Publishing Company (1962), 133135.

[SST] J. Spencer, E. Szemerédi and W. T. Trotter, Unit Distances in the Euclidean Plane, Graph Theory and Combinatorics, Academic Press, London (1984), 253-278.

[Th] T. Thiele, Point Sets with Distinct Slopes or Lengths, preprint, (1993).

[Vg] R. C. Vaughan, The Hardy-Littlewood Method, Cambridge University Press (1981), 1-25.

[Wi] B. M. Wilson, Proofs of Some Formulæ Enunciated by Ramanujan, Proceedings London Mathematical Society 21 (1923), 235-255. 